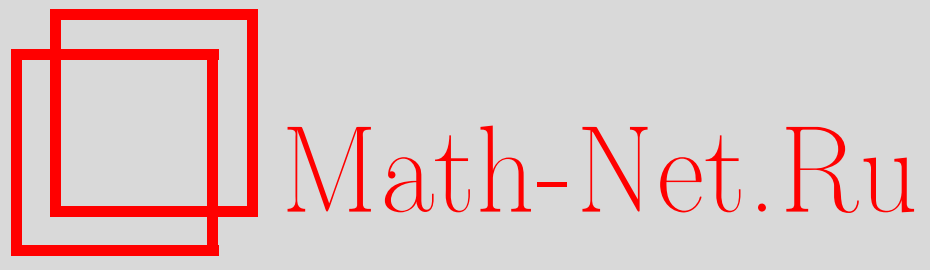

Я. И. Белопольская, Стохастическая интерпретация квазилинейных параболических систем с кросс-диффузией, Теория вероятн. и ее примен., 2016, том 61, выпуск 2, 268-299

DOI: https://doi.org/10.4213/tvp5056

Использование Общероссийского математического портала Math-Net.Ru подразумевает, что вы прочитали и согласны с пользовательским соглашением http: //www . mathnet.ru/rus/agreement

Параметры загрузки:

IP : 54.89 .56 .158

26 апреля 2023 г., 16:06:54

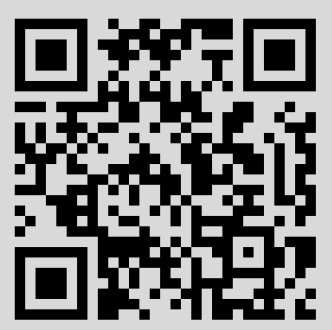




\section{СТОХАСТИЧЕСКАЯ ИНТЕРПРЕТАЦИЯ КВАЗИЛИНЕЙНЫХ ПАРАБОЛИЧЕСКИХ СИСТЕМ С КРОСС-ДИФФУЗИЕЙ}

В работе построена вероятностная интерпретация обобщенного решения задачи Коши для сильно связанной системы квазилинейных параболических уравнений. С этой целью выведена система стохастических уравнений, решение которой позволяет получить вероятностное представление решения исходной задачи и изучить свойства этого решения. Предложенная конструкция вероятностного представления решения задачи Коши существенно опирается на результаты теории стохастических потоков.

Ключевые слова и фразы: стохастические уравнения, системы квазилинейных параболических уравнений, классические и обобщенные решения задачи Коши, стохастические потоки.

Введение Системы квазилинейных параболических уравнений возникают в качестве математических моделей динамики ряда биологических систем, в частности, процессов хемотаксиса, динамики роста клеток при контактном ингибировании, динамики взаимодействующих популяций, а также ряда других. В общем случае такие системы имеют вид

$u_{t}^{q}=\operatorname{div}\left(F^{q m}(x, u) \nabla u^{m}\right)+a^{q m}(x, u, \nabla u) \nabla u^{m}+f^{q}(x, u), \quad q, m=1, \ldots, d_{1}$.

Здесь и ниже, если не оговорено противное, будет предполагаться суммирование по всем повторяющимся индексам и приняты обозначения $u_{t}=\partial u / \partial t, \nabla u=\left(\nabla_{1} u, \ldots, \nabla_{d} u\right), \nabla_{i} u=\partial u / \partial x_{i}, i=1, \ldots, d$.

Системы параболических уравнений, возникающие в качестве моделей динамики взаимодействующих популяций, называют сильно связанными системами и их изучению посвящено много работ как общего [1], так и прикладного характера [2], [3]. Обзоры современных результатов по исследованию краевых задач для таких систем можно найти, например, в работах [4], [5].

*Петербургский Государственный Архитектурно-Строительный Университет, 190005, Санкт-Петербург, уаnа@yb1569.spb.edu

1) Работа выполнена при поддержке РФФИ (грант № 15-01-01453). 
Один из важных примеров систем такого вида возникает при изучении динамики взаимодействующих популяций в пространственно неоднородной среде с учетом их взаимного влияния. Напомним, что классическая модель Лотка-Вольтерра динамики взаимодействующих популяций в пространственно однородной системе представляет собой систему нелинейных обыкновенных дифференциальных уравнений. Учет самодиффузии в пространственно неоднородном случае приводит к системе параболических уравнений, не имеющих непостоянных стационарных состояний, что противоречит существованию наблюдаемых биологических структур. Неоднородные стационарные состояния возникают, если принимать во внимание наличие кросс-диффузии; такая модель была предложена в работе [6] для описания динамики двух взаимодействующих популяций. В рамках этой модели, называемой моделью SKT, предполагается, что основной причиной рассеяния является миграция во избежание перенаселения, а не простая диффузия, обусловленная случайным блужданием.

Задача Коши для системы параболических уравнений с кроссдиффузией, соответствующая модели SKT, имеет следующий вид:

$$
\begin{cases}u_{t}=\Delta\left[\left(\alpha_{1}+\alpha_{11} u+\alpha_{12} v\right) u\right]+u\left(a_{1}-b_{1} u-c_{1} v\right), & u(0, x)=u_{0}(x), \\ v_{t}=\Delta\left[\left(\alpha_{2}+\alpha_{21} u+\alpha_{22} v\right) v\right]+v\left(a_{2}-b_{2} u-c_{2} v\right), & v(0, x)=u_{v}(x) .\end{cases}
$$

Здесь и ниже $\operatorname{div} \nabla u=\Delta u$ - оператор Лапласа, $\alpha_{q}, \alpha_{q m}, a_{q}, b_{q}, c_{q}-$ неотрицательные константы, $q, m=1,2, u$ и $v$ - плотности популяций.

Модель SKT сразу привлекла внимание математиков. Одними из первых были работы [2], [3], в которых были исследованы классические (дважды дифференцируемые) стационарные решения этой системы при различных дополнительных предположениях о коэффициентах.

Обобщенные нестационарные решения задачи (0.1) были определены в работе [1], где также были получены и достаточные условия глобального существования таких решений. При этом вопрос о глобальном существовании локального по времени решения системы (0.1) был сведен к задаче получения априорных оценок в соболевском пространстве $W^{1, p}$. Точнее говоря, было показано следующее. Пусть $T$ - максимальное время существования локального решения, тогда, если локальное решение ограничено в $W^{1, p}$ равномерно по $t \in(0, T)$ при $p>d$, где $d$ - размерность пространственной переменной, то существует глобальное решение.

В соответствии с определением, сформулированным в работе [1], обобщенныл решением системь (0.2) называется пара функций $u, v$, удовлетворяющих следующим условиям:

(1) $u, v \in L_{\mathrm{loc}}^{\infty}\left([0, \infty) ; L^{\infty}\left(\mathbf{R}^{d}\right)\right), u, v \geqslant 0$;

(2) $\nabla(u+v) \in L_{\text {loc }}^{2}\left((0, \infty) \times \mathbf{R}^{d}\right)$; 
(3) Для любой пробной функции $h \in C_{c}^{\infty}\left(\mathbf{R}^{d}\right)$ с компактным носителем справедливы интегральные тождества:

$$
\left\{\begin{array}{c}
\left\langle u_{t}, h\right\rangle+B^{u}[u, v, h](t)-\left\langle\left(a_{1}-b_{1} u-c_{1} v\right) u, h\right\rangle=0, \\
\left\langle v_{t}, h\right\rangle+B^{v}[u, v, h](t)-\left\langle\left(a_{2}-b_{2} u-c_{2} v\right) v, h\right\rangle=0 .
\end{array}\right.
$$

где

$$
\begin{aligned}
& B^{u}[u, v, h](t)=\frac{1}{2} \int_{\mathbf{R}^{d}} \nabla\left[u(t, x)\left(\alpha_{1}+\alpha_{11} u(t, x)+\alpha_{12} v(t, x)\right)\right] \cdot \nabla h(x) d x, \\
& B^{v}[u, v, h](t)=\frac{1}{2} \int_{\mathbf{R}^{d}} \nabla\left[v(t, x)\left(\alpha_{2}+\alpha_{21} u(t, x)+\alpha_{22} v(t, x)\right)\right] \cdot \nabla h(x) d x .
\end{aligned}
$$

Здесь и ниже обозначение $\langle u, h\rangle=\int_{\mathbf{R}^{d}} u(x) h(x) d x$ будет использовано либо как обозначение для скалярного произведение в гильбертовом пространстве (например, в $L^{2}\left(\mathbf{R}^{d}\right)$ или $H^{1}\left(\mathbf{R}^{d}\right)$ ), либо для обозначения спаривания между двойственными пространствами (например, соболевскими пространствами $H^{k}\left(\mathbf{R}^{d}\right)$ и $H^{-k}\left(\mathbf{R}^{d}\right)$ для натурального числа $\left.k\right)$, а $x \cdot y=\sum_{k=1}^{d} x_{k} y_{k}$ обозначает скалярное произведение в $\mathbf{R}^{d}$.

Целью этой работы является построение вероятностных представлений обобщенного решения задачи (0.2) и развитие вероятностного подхода к исследованию задачи Коши для систем такого вида. При этом заметим, что предложенная в этой работе вероятностная интерпретация системы (0.2) мотивирована другим определением обобщенного решения, которое также используется в теории уравнений в частных производных (см., например, [5]).

Насколько нам известно, впервые попытки построить вероятностные представления решения задачи Коши для полностью недиагональных систем были предприняты в [7]-[9]. В этих работах было замечено, что интерпретируя рассматриваемую систему как двойственную систему уравнений, можно связать с ней соответствующие случайные процессы и показать, что обобщенное решение задачи Коши вида (0.1) (для некоторых частных случаев) можно получить в виде средних на траекториях соответствующих случайных процессов, используя результаты теории стохастических потоков Куниты [10]-[12] и соответствующие результаты работ Далецкого и автора [13], [14], а также работ [15], [16], в которых были сформулированы вероятностные методы построения обобщенных решений задачи Коши для семилинейных параболических уравнений и систем с диагональной главной частью (без кросс-диффузии). Отметим еще альтернативный вероятностный подход к выводу уравнений вида (0.2), основанный на моделировании популяционной динамики с помощью мерозначных марковских процессов [17], [18]. При этом рассматриваемая параболическая система с кросс-диффузией возникает как предел (при неограниченном увеличении числа частиц) уравнений, ко- 
торым подчиняется плотность распределения системы взаимодействующих частиц, моделируемая соответствующей системой стохастических уравнений.

Далее структура статьи имеет следующий вид. В п. 1 мы приведем стохастические уравнения, описывающие диффузионные процессы, связанные с уравнениями, двойственными (в смысле теории обобщенных функций) к уравнениям рассматриваемой системы. В п. 2 будут приведены в удобной для наших целей форме необходимые результаты теории стохастических потоков. В случае отсутствия кросс-диффузионных членов и при априорном предположении о существовании и единственности регулярного обобщенного решения исходной задачи Коши будет построено вероятностное представление этого решения. В п.3 мы откажемся от априорного предположения о существовании решения исходной задачи. Замкнутая система стохастических уравнений для случайных процессов, ассоциированных с рассматриваемой параболической системой без кросс-диффузии, будет исследована независимо от исходной задачи Коши и будут сформулированы условия, при которых у этой системы существует единственное решение (возможно, на малом временном интервале). В заключение этого параграфа будет показано, что, решив рассматриваемую стохастическую задачу, мы одновременно построим искомое обобщенное (возможно, локальное по времени) решение исходной задачи Коши. В последнем пункте будет приведено вероятностное представление решения задачи Коши для параболической системы с кросс-диффузией и приведена стохастическая интерпретация этой системы. Здесь будут приведены стохастические уравнения, определяющие случайные процессы, участвующие в построении вероятностного представления обобщенного решения задачи Коши, и показано, что, при априорном предположении о существовании и единственности регулярного обобщенного решения задачи Коши для исходной параболической системы, решения этих стохастических уравнений существуют и позволяют строить требуемые вероятностные представления этого обобщенного решения.

\section{1. Вероятностная интерпретация решения задачи Коши} для двойственной системы параболических уравнений.

Рассмотрим задачу Коши для системы параболических уравнений

$$
\begin{gathered}
u_{t}^{1}=\frac{1}{2} \Delta\left(u^{1}\left(\alpha_{1}+\alpha_{11} u^{1}+\alpha_{12} u^{2}\right)\right)+u^{1}\left(a_{1}-b_{1} u^{1}-c_{1} u^{2}\right), \\
u^{1}(0, x)=u_{0}^{1}(x), \\
u_{t}^{2}=\frac{1}{2} \Delta\left(u^{2}\left(\alpha_{2}+\alpha_{21} u^{1}+\alpha_{22} u^{2}\right)\right)+u^{2}\left(a_{2}-b_{2} u^{1}-c_{2} u^{2}\right), \\
u^{2}(0, x)=u_{0}^{2}(x) .
\end{gathered}
$$


Для определения обобщенного решения задачи Коши (1.1), (1.2) нам понадобится ряд функциональных пространств.

Пусть $\mathscr{D}$ - пространство Шварца, т.е. пространство бесконечно дифференцируемых скалярных функций на $\mathbf{R}^{d}$ с компактными носителями, и $\mathscr{D}^{\prime}$ - его сопряженное. Обозначим

$$
\langle u, h\rangle=\int_{\mathbf{R}^{d}} u(x) h(x) d x \quad \forall u \in \mathscr{D}^{\prime}, \quad h \in \mathscr{D} .
$$

Если $u \in \mathbf{R}^{d}$ и $h \in \mathbf{R}^{d}$ - векторные функции, компоненты которых принадлежат $\mathscr{D}$, то

$$
\langle u, h\rangle=\int_{\mathbf{R}^{d}} u(x) \cdot h(x) d x .
$$

Пусть $Z$ обозначает множество всех положительных целых чисел, $k \in Z$ и $H^{k} \equiv H^{k}\left(\mathbf{R}^{d}\right)=\left\{v \in L^{2}\left(\mathbf{R}^{d}\right): \nabla^{\alpha} v \in L^{2}\left(\mathbf{R}^{d}\right)\right\}$ обозначает множество всех вещественных функций $v$, определенных на $\mathbf{R}^{d}$ и квадратично интегрируемых вместе со своими (обобщенными) производными $D^{\alpha} v$ порядка $\alpha,|\alpha|=\sum_{i} \alpha_{i} \leqslant k$, где

$$
D^{\alpha} v=\frac{\partial^{\alpha_{1}}}{\partial x_{1}^{\alpha_{1}}} \cdots \frac{\partial^{\alpha_{n}}}{\partial x_{n}^{\alpha_{n}}} v
$$

и $\sum_{n=1}^{d} \alpha_{n}=|\alpha|=k$. При этом $H^{k}$ - это гильбертово пространство с нормой

$$
\|v\|_{k}=\left(\sum_{|\alpha| \leqslant k} \int_{\mathbf{R}^{d}}\left\|D^{\alpha} v(x)\right\|^{2} d x\right)^{1 / 2} .
$$

Обозначим $H^{-k}$ пространство, двойственное к $H^{k}$, с нормой

$$
\|u\|_{H^{-k}}=\sup \left\{\left|\langle u, h\rangle_{k}\right|: h \in H_{0}^{k},\|h\|_{H_{0}^{k}} \leqslant 1\right\}
$$

$H_{0}^{k}$ - подмножество функций из $H^{k}$ с компактными носителями и пусть $\mathscr{W}^{k}$ - пространство квадратично-интегрируемых функций $h$, заданных на $[0, T] \times \mathbf{R}^{d}, T>0$,

$$
\mathscr{W}^{k}=\left\{h \in L^{2}\left([0, T] \times \mathbf{R}^{d}\right) ; \nabla^{\alpha} h \in L^{2}\left([0, T] \times \mathbf{R}^{d}\right),|\alpha| \leqslant k\right\},
$$

с нормой

$$
\|h\|_{k}=\left(\int_{0}^{T} \int_{\mathbf{R}^{d}}\left[\sum_{|\alpha| \leqslant k}\left\|D^{\alpha} h(\theta, x)\right\|^{2}\right] d x d \theta\right)^{1 / 2} .
$$

Будем говорить, что пара положительных функций $u^{1}(t), u^{2}(t) \in H^{1}$ является обобщенным решением задачи Коши для системы (1.1), (1.2), 
если для любой $h \in H^{1}$ и п.в. $0 \leqslant t \leqslant T$ справедливы интегральные тождества вида (0.3). Ниже полезным окажется несколько иное определение обобщенного решения системы параболических уравнений.

Предположим, что пробные функции $h: \mathbf{R}_{+} \times \mathbf{R}^{d} \rightarrow \mathbf{R}$ принадлежат пространству $\mathscr{H}_{0}^{1,2}$, где $\mathscr{H}_{0}^{1,2}$ - пространство вещественных функций с компактными носителями, дифференцируемых по $t \in(0, \infty)$ и дважды дифференцируемых по $x \in \mathbf{R}^{d}$.

Будем говорить, что задача Коши (1.1), (1.2) имеет слабое обобщенное решение $u^{1}, u^{2}$, если для любой пробной функции $h \in \mathscr{H}_{0}^{1,2}\left([0, t] \times \mathbf{R}^{d}\right)$ справедливы интегральные тождества

$$
\begin{aligned}
& \int_{\mathbf{R}^{d}} u^{q}(t, x) h(t, x) d x \\
& =\int_{0}^{t} \int_{\mathbf{R}^{d}} u^{q}(\theta, x)\left\{h_{\mathbf{R}^{d}} u^{q}(\theta, x) h(0, x) d x\right. \\
& \left.\quad+\frac{1}{2}\left[\alpha_{q}+\alpha_{q 1} u^{1}(\theta, x)+\alpha_{q 2} u^{2}(\theta, x)\right] \Delta h(\theta, x)\right\} d x d \theta \\
& \quad+\int_{0}^{t} \int_{\mathbf{R}^{d}} u^{q}(\theta, x) m_{u}^{q}(\theta, x) h(\theta, x) d x d \theta, \quad q=1,2,
\end{aligned}
$$

где

$$
m_{u}^{q}(x)=a_{q}-b_{q} u^{1}(x)-c_{q} u^{2}(x), \quad u=\left(u^{1}, u^{2}\right) .
$$

Введем дополнительно обозначения

$$
\mathscr{L}_{0}^{q} u^{q}=\frac{1}{2} \Delta\left[u^{q}\left(\alpha_{q}+\alpha_{q 1} u^{1}+\alpha_{q 2} u^{2}\right)\right], \quad \mathscr{L}_{u}^{q}=\mathscr{L}_{0}^{q}+m_{u}^{q}, \quad q=1,2,
$$

И

$$
M_{u}^{q}(x)=\sqrt{\alpha_{q}+\alpha_{q 1} u^{1}(x)+\alpha_{q 2} u^{2}(x)} .
$$

Тогда интегральные тождества можно записать в виде

$$
\begin{gathered}
\int_{0}^{t} \int_{\mathbf{R}^{d}}\left[u_{\theta}^{q}(\theta, x)-\mathscr{L}_{0}^{q} u^{q}(\theta, x)-m_{u}^{q}(\theta, x) u^{q}(\theta, x)\right] h(\theta, x) d x d \theta \\
=\int_{\mathbf{R}^{d}} u^{q}(t, x) h(t, x) d x-\int_{\mathbf{R}^{d}} u^{q}(0, x) h(0, x) d x \\
-\int_{0}^{t} \int_{\mathbf{R}^{d}} u^{q}(\theta, x)\left[h_{\theta}(\theta, x)+\frac{1}{2}\left[M_{u}^{q}\right]^{2} \Delta h(\theta, x)\right. \\
\left.+m_{u}^{q}(\theta, x) h(\theta, x)\right] d x d \theta=0 .
\end{gathered}
$$

Пусть $0 \leqslant s \leqslant \theta \leqslant t$. Для того, чтобы построить случайные процессы, связанные с обобщенным решением задачи Коши (1.1), (1.2), предположим, что существует единственное ограниченное решение $u=$ $\left(u^{1}, u^{2}\right)$ этой задачи, $u^{q}(t) \in C^{2}\left(\mathbf{R}^{d}\right), q=1,2$, удовлетворяющее условию 
$\inf _{x} u^{q}(t, x) \geqslant 0$, и рассмотрим задачу Коши для параболических уравнений

$$
\begin{gathered}
h_{s}^{1}+\frac{1}{2}\left[M_{u}^{1}\right]^{2} \Delta h^{1}+m_{u}^{1} h^{1}=0, \quad h^{1}(t, y)=h_{0}^{1}(y), \quad 0 \leqslant s \leqslant t, \\
h_{s}^{2}+\frac{1}{2}\left[M_{u}^{q}\right]^{2} \Delta h^{2}+m_{u}^{2} h^{2}=0, \quad h^{2}(t, y)=h_{0}^{2}(y),
\end{gathered}
$$

двойственную (в смысле интегральных тождеств (1.7)) к системе (1.1), (1.2).

При сформулированных выше априорных предположениях, если $h_{0}^{q} \in C^{2}\left(\mathbf{R}^{d}\right)$, то существует единственное классическое решение $h(s, x)=\left(h^{1}(s, x), h^{2}(s, x)\right)$ задачи Коши $(1.8),(1.9)$, допускающее соответствующее вероятностное представление.

Для того, чтобы построить это представление, введем в рассмотрение вероятностное пространство $(\Omega, \mathscr{F}, \mathbf{P})$ и заданный на нем стандартный винеровский процесс $w(t) \in \mathbf{R}^{d}, q=1,2$. Обозначим $\mathscr{F}_{t} \subset \mathscr{F}$ поток $\sigma$-алгебр, порожденный процессом $w(t)$.

Рассмотрим систему стохастических дифференциальных уравнений (СДУ)

$$
d \xi^{q}(\theta)=M_{u}^{q}\left(\xi^{q}(\theta)\right) d w(\theta), \quad \xi^{q}(s)=y, \quad q=1,2 .
$$

Поскольку в силу априорного предположения $u^{q} \in C^{2}\left(\mathbf{R}^{d}\right)$, и, следовательно, коэффициенты $M_{u}^{q}$ в $(1.10)$ являются ограниченными липшицевыми функциями, то существование и единственность решения (1.10) вытекают из общей теории СДУ. Другими словами, справедливо следующее утверждение.

Tеорема 1.1. Пусть $\left.\inf _{x} u^{q}(\theta, x)\right) \geqslant 0 u \nabla u^{q}$ - ограниченнье функиии, удовлетворяющие условию Липшииа (по $x)$, тогда СДУ (1.10) имеет единственное сильное решение $\xi_{s, y}^{q}(\theta)$ для всех $0 \leqslant s \leqslant \theta \leqslant t u$ прочессыл $\xi^{q}(\theta) \equiv \xi_{s, y}^{q}(\theta)$ обладают марковским свойством.

Вероятностное представление решения $h^{q}(s, y)$ задачи Коши (1.8), (1.9) нетрудно получить, если ввести дополнительно в рассмотрение случайные процессы

$$
\eta^{q}(t)=\exp \left\{\int_{s}^{t} m_{u}^{q}\left(\xi^{q}(\theta)\right) d \theta\right\} .
$$

Теорема 1.2. Если выполнень условия теоремь 1.1, функиии $u^{1}, u^{2}$ принадлежат классу $C^{1,2}\left([0, T] \times \mathbf{R}^{d}\right) u h \in C_{0}^{2}\left(\mathbf{R}^{d}\right)$, то пара функций $h^{1}, h^{2}$, заданная соотношениями

$$
h^{q}(s, y)=\mathbf{E}\left[\exp \left\{\int_{s}^{t} m_{u}^{q}\left(\xi_{s, y}^{q}(\theta)\right) d \theta h_{0}^{q}\left(\xi_{s, y}^{q}(t)\right)\right\}\right], \quad q=1,2
$$

является единственным классическим решением системь (1.8), (1.9). 
В силу условий теоремы 1.2 , ее утверждение вытекает из результатов классической теории стохастических дифференциальных уравнений, и соотношение (1.11) - это классическая формула Фейнмана-Каца.

3 а м е ч а н и е. Рассмотрим СДУ вида

$$
\left.d \gamma^{q}(\theta)=a_{u}^{q}\left(\gamma^{q}(\theta)\right) d \theta+M_{u}^{q}\left(\gamma^{q}(\theta)\right)\right) d w(\theta), \quad \gamma^{q}(s)=y, \quad q=1,2,
$$

где, как и выше, $u^{q} \in C^{1,2}\left([0, T] \times \mathbf{R}^{d}\right)$ - заданная функция и $a^{q}(x, u) \in$ $\mathbf{R}^{d}$ - функция класса $C^{2}\left(\mathbf{R}^{d} \times \mathbf{R}^{2}\right)$. Тогда, как нетрудно проверить, функция

$$
\bar{h}^{q}(s, y)=\mathbf{E}\left[\exp \left\{\int_{s}^{t} m_{u}^{q}\left(\gamma_{s, y}^{q}(\theta)\right) d \theta\right\} h_{0}^{q}\left(\gamma_{s, y}^{q}(t)\right)\right], \quad q=1,2,
$$

является единственным классическим решением задачи Коши

$$
\bar{h}_{s}^{q}+a_{u}^{q} \cdot \nabla \bar{h}^{q}+\frac{1}{2}\left[M_{u}^{q}\right]^{2} \Delta \bar{h}^{1}+m_{u}^{q} \bar{h}^{q}=0, \quad \bar{h}^{q}(t, y)=h_{0}^{q}(y), \quad 0 \leqslant s \leqslant t .
$$

Если $M^{q}(u) \equiv 0$, то (1.14) описывает гиперболическую систему, для которой рассматривается обратная задача Коши с данными на правом конце временного интервала. Стохастическое уравнение (1.12) при этом превращается в уравнение $d \gamma_{0}^{q}(\theta) / d \theta=a_{u}^{q}\left(\gamma_{0}^{q}(\theta)\right)$, совпадающее с уравнением для характеристик рассматриваемой гиперболической системы, а соотношение (1.13), приобретает вид $\bar{h}_{0}^{q}(s, y)=$ $\exp \left\{\int_{s}^{t} m_{u}^{q}\left(\gamma_{0}^{q}(\theta)\right) d \theta\right\} h_{0}^{q}\left(\gamma_{0}^{q}(t)\right)$ и может быть получено методом характеристик.

Для того, чтобы перейти от изучения решения задачи Коши для системы (1.8), (1.9) к построению обобщенного решения исходной системы (1.1), (1.2), нам понадобится ряд результатов из теории стохастических потоков и теории двойственности для марковских процессов.

\section{2. Стохастические потоки и решения задачи Коши для па-} раболических уравнений и систем. Как уже упоминалось выше, теория стохастических уравнений представляет собой обобщение теории обыкновенных дифференциальных уравнений и соответственно решения СДУ для линейных и нелинейных параболических уравнений и систем играют ту же роль, что и характеристики в теории линейных и нелинейных гиперболических уравнений и систем. Выше эта аналогия была проиллюстрирована на примере решения обратной задачи Коши.

Эту аналогию можно сохранить и при переходе к рассмотрению прямой задачи Коши. Рассмотрим в качестве примера задачу Коши для гиперболической системы Хопфа

$$
u_{t}+u \cdot \nabla u=0, u(0, x)=u_{0}(x) \in \mathbf{R}^{d} .
$$


Решая эту задачу методом характеристик, рассмотрим систему ОДУ

$$
\begin{gathered}
\frac{d u}{d t}=0, \quad u(0)=u_{0}(y) \in \mathbf{R}^{d}, \\
\frac{d x}{d t}=u, \quad x(0)=y \in \mathbf{R}^{d},
\end{gathered}
$$

решение которой имеет вид

$$
u(t, x)=u_{0}(y), \quad x=y+u_{0}(y) t,
$$

где функция $u$ зависит от $t$ и $x$, поскольку $y=y(t, x)$ рассматривается как неявная функция от $t$ и $x$. Заметим, что отображение $\phi_{0, t}: y \rightarrow x$, порожденное решением $x(t)=\phi_{0, t}(y)$ задачи Коши (2.3), обратимо на интервале $\left[0, t_{c}\right)$, на котором обратима матрица $\left[\nabla u_{0}(y)\right]^{-1}$. Обозначим $\psi_{t, 0}: x \rightarrow y$ отображение, обратное по отношению к $\phi_{0, t}$, для которого справедливо соотношение $\phi_{0, t} \circ \psi_{0, t}(x)=x$. Тогда, обозначив $\widehat{x}(s)=$ $x(t-s)$, получим $u(t, x)=u_{0}\left(\psi_{0, t}(x)\right)=u_{0}\left(\widehat{x}_{0, x}(t)\right)$.

Рассмотрение задачи Коши для системы Хопфа с диссипацией

$$
u_{t}+u \cdot \nabla u=-c u, \quad u(0, x)=u_{0}(x),
$$

где $c$ - положительная константа, приводит к модифицированной системе ОДУ,

$$
\frac{d u}{d t}=-c u, \quad u(0)=u_{0}(y), \quad \frac{d x}{d t}=u, \quad x(0)=y,
$$

задающей ее характеристики. Решение этой задачи на интервале $\left[0, t_{c}\right]$, на котором обратим градиент матрицы $\nabla u_{0}$, можно представить в виде

$$
u(t, x)=u_{0}\left(\widehat{x}_{0, x}(t)\right) e^{-c t}, \quad x=y+\frac{1}{c} u_{0}\left(\widehat{x}_{0, x}(t)\right)\left(1-e^{-c t}\right) .
$$

Для того, чтобы распространить этот подход на теорию параболических систем, перейдем от системы Хопфа (2.1) к системе Бюргерса с вязкостью, что приведет к рассмотрению следующей задачи Коши

$$
u_{t}+u \cdot \nabla u=\frac{1}{2} \sigma^{2} \Delta u, \quad u(0, x)=u_{0}(x),
$$

где $\sigma$ - положительная константа.

Отметим, что аналогом решения задачи Коши для гиперболического уравнения, построенного с помощью метода характеристик, является вероятностное представление решения задачи Коши (2.5) с помощью диффузионных процессов, удовлетворяющих соответствующим СДУ. Для того, чтобы построить такое представление, рассмотрим СДУ

$$
d \xi(\theta)=u(\theta, \xi(\theta)) d \theta+\sigma d w(\theta), \quad \xi(0)=y, \quad 0 \leqslant s \leqslant \theta \leqslant t .
$$


Задать функцию $u(t, x)$ так, чтобы получить замкнутую систему соотношений и, в то же время, чтобы полученное соотношение можно было интерпретировать как решение задачи (2.5), можно двумя способами. Один из них состоит в том, чтобы вместо того, чтобы решать прямую задачу Коши (2.5) относительно функции $u(t, x)$, ввести в рассмотрение функцию $v(s, x)=u(t-s, x)$. При этом, очевидно, задача Коши (2.5) относительно $u$ будет сведена к задаче Коши

$$
v_{s}-v \cdot \nabla v+\frac{1}{2} \sigma^{2} \Delta v=0, \quad v(t, x)=u_{0}(x)
$$

относительно функции $v$. Как следует из работ [13], [14], классическое решение задачи Коши (2.7) можно построить, решив следующую стохастическую систему

$$
\begin{gathered}
d \xi(\theta)=-v(t-\theta, \xi(\theta)) d \theta+\sigma d w(\theta), \quad \xi(s)=x, \\
v(s, x)=\mathbf{E}\left[u_{0}\left(\xi_{s, x}(t)\right] .\right.
\end{gathered}
$$

Если начальная функция $u_{0}(x)$ достаточно гладкая, то функция $v(s, x)$ вида (2.8) определяет классическое решение задачи Коши (2.7) (возможно, локальное по времени) и, соответственно, функция $u(t, x)$ удовлетворяет задаче Коши (2.5).

Второй подход представляет собой обобщение подхода к решению гиперболической задачи, описанного в начале этого параграфа, и может быть сформулирован следующим образом.

Пусть $u(t, x)$ — дважды дифференцируемая ограниченная функция. Тогда, в силу результатов классической теории стохастических дифференциальных уравнений (СДУ), решение (2.6) существует, единственно и дважды дифференцируемо по начальным условиям. При этом случайный процесс $\xi(t)$, удовлетворяющий (2.6), обладает марковским свойством и задает стохастический поток $\phi_{0, t}: y \mapsto \xi_{0, y}(t)$. Обозначим $\psi_{t, 0}$ обратный по времени стохастический поток, удовлетворяющий условию $\psi_{t, 0} \circ \phi_{0, t}(y)=y$ и пусть $\widehat{\xi}(\theta)=\xi(t-\theta)$ - обращенный по времени процесс, порождающий поток $\psi_{t, 0}$. Рассмотрим систему стохастических уравнений

$$
\begin{gathered}
d \widehat{\xi}(\theta)=u(\theta, \widehat{\xi}(\theta)) d \theta+\sigma d \widetilde{w}(\theta), \quad \widehat{\xi}(0)=x, \\
u(t, x)=\mathbf{E}\left[u_{0}\left(\widehat{\xi}_{0, x}(t)\right)\right],
\end{gathered}
$$

где $\widetilde{w}(\theta)=w(t-\theta)-w(t)$. Эта система представляет собой замкнутую систему уравнений и, как следует из результатов [15], при определенных условиях на $u_{0}$, ее решение существует на некотором интервале $[0, T]$.

Возвращаясь к задачам, рассмотренным в п.1, воспользуемся вторым подходом для того, чтобы построить соответствующие процессы $\xi^{q}(t) \in \mathbf{R}^{d}, q=1,2$, и обращенные во времени процессы $\widehat{\xi}^{q}(\theta)=\xi^{q}(t-\theta) \in$ 
$\mathbf{R}^{d}$, которые позволили бы получить вероятностное представления решения прямой задачи Коши для системы (1.1), (1.2). При этом нам понадобится ряд результатов теории стохастических потоков Куниты [10].

В этом и следующем параграфах мы предположим, что $\alpha_{12}=$ $\alpha_{21}=0$, и существует единственное ограниченное регулярное (дважды дифференцируемое по $x$ ) обобщенное положительное решение $u(t, x)=$ $\left(u^{1}(t, x), u^{2}(t, x)\right)$ задачи $(1.1),(1.2)$. Рассмотрим систему стохастических уравнений

$$
d \xi^{q}(\theta)=-M_{u}^{q}\left(\xi^{q}(\theta)\right) d w(\theta), \quad \xi^{q}(s)=y, \quad q=1,2 .
$$

При сделанном выше априорном предположении мы находимся в рамках теории Куниты, что позволяет воспользоваться результатами работ [11], [12].

Для заданных процессов $\xi^{q}(t) \in \mathbf{R}^{d}$ и $\widehat{\xi}^{q}(\theta)=\xi^{q}(t-\theta) \in \mathbf{R}^{d}$ обозначим $\phi_{\theta, t}^{q}: \mathbf{R}^{d} \rightarrow \mathbf{R}^{d}, \psi_{\theta, t}^{q}: \mathbf{R}^{d} \rightarrow \mathbf{R}^{d}$ порожденные ими стохастические потоки, т.е. $\xi_{\theta, x}^{q}(t)=\phi_{\theta, t}^{q}(x), \widehat{\xi}_{t, y}^{q}(\theta)=\psi_{\theta, t}^{q}(y)$. Как и выше, предположим, что $u^{1}, u^{2}$ - дифференцируемые по $x$ ограниченные квадратично интегрируемые функции, удовлетворяющие в слабом смысле задаче $(1.1),(1.2)$. Тогда процессы $\xi^{q}(t)$, удовлетворяющие $(2.1)$, дифференцируемы (в среднем квадратичном) по начальным данным.

Пусть $\mathbf{J}_{s, y}^{q}(t)=\nabla \xi_{s, y}^{q}(t)$ - матрица Якоби стохастического отображения $\phi_{s, t}^{q}: \mathbf{R}^{d} \rightarrow \mathbf{R}^{d}$. Для краткости мы будем иногда обозначать $\mathbf{J}_{s, y}^{q}(t) \equiv \mathbf{J}^{q}(s, t), 0 \leqslant s \leqslant t$. Пусть $J_{s, y}^{q}(t)=\operatorname{det} \mathbf{J}_{s, y}^{q}(t) \equiv J_{s, t}^{q}$.

Матрица Якоби $\mathbf{J}^{q}(s, t)$ удовлетворяет линейному СДУ

$$
d \mathbf{J}^{q}(s, \theta)=-\mathbf{J}^{q}(s, \theta) \nabla M_{u}^{q}\left(\xi_{s, y}^{q}(\theta)\right) \cdot d w(\theta), \quad \mathbf{J}^{q}(s, s)=I,
$$

где $I$ - единичная матрица, $s \leqslant \theta \leqslant t$. Нетрудно проверить, что процессы $\widehat{\xi}^{q}(\theta)=\xi^{q}(t-\theta)$ также дифференцируемы по начальным данным и из соотношений

$$
\widehat{\mathbf{J}}^{q}(\theta, t) \mathbf{J}^{q}(\theta, t)=\mathbf{J}^{q}(\theta, t) \widehat{\mathbf{J}}^{q}(\theta, t)=I, \quad d\left[\widehat{\mathbf{J}}^{q}(\theta, t) \mathbf{J}^{q}(\theta, t)\right]=0,
$$

и формулы Ито при этом вытекает, что $\widehat{\mathbf{J}}^{q}(\theta, t)=\nabla \widehat{\xi}^{q}(\theta)$ удовлетворяет СДУ

$$
d \widehat{\mathbf{J}}^{q}(\theta, t)=\widehat{\mathbf{J}}^{q}(\theta, t) \kappa^{q}(\theta) d \theta+\widehat{\mathbf{J}}^{q}(\theta, t) \nabla M_{u}^{q}\left(\xi^{q}(\theta)\right) \cdot d w(\theta), \quad \widehat{\mathbf{J}}^{q}(t, t)=I,
$$

где

$$
\kappa^{q}(\theta)=\left[\nabla M_{u}^{q}\left(\xi^{q}(\theta)\right)\right]^{2} .
$$

Заметим, что $\widehat{\mathbf{J}}_{\theta, t}^{g}(\omega)>0$.

Можно показать (см. [14], [19]), что случайный процесс $\widehat{\xi}^{q}(\tau)=\xi^{q}(t-$ $\tau)$ удовлетворяет стохастическому уравнению

$$
\widehat{\xi}^{q}(\tau)=\xi^{q}(t)+\int_{0}^{\tau} \beta_{u}^{q}\left(\widehat{\xi}^{q}(\theta)\right) d \theta+\int_{0}^{\tau} M_{u}^{q}\left(\widehat{\xi}^{q}(\theta)\right) d \widetilde{w}(\theta)
$$


где, как и выше, $\widetilde{w}(\theta)=w(t-\theta)-w(t), 0 \leqslant \tau \leqslant t$ и $\beta_{u}^{q}(x)=M_{u}^{q}(x) \nabla M_{u}^{q}(x)$.

Случайный процесс в пространстве обобщенных функций $\mathscr{D}^{\prime}$ или в соболевском пространстве $H^{-k}$ можно задать, определив композицию обобщенной функции со стохастическим потоком. Для функции $h$ из пространства Шварца $\mathscr{D}$ и обобщенной функции $g$ из двойственного пространства $\mathscr{D}^{\prime}$ определим композицию $g$ с потоком $\psi_{\theta, t}$, порожденным процессом $\widehat{\xi}^{q}(\theta)$, с помощью соотношения $\left\langle g \circ \psi_{\theta, t}, h\right\rangle=\left\langle g, h \circ \phi_{\theta, t} J_{\theta, t}\right\rangle$. Правая часть последнего равенства, как нетрудно проверить, задает непрерывный линейный функционал на $\mathscr{D}$, если $\phi_{s, t}(x)$ - бесконечно дифференцируемое отображение. Если же $\phi_{s, t}-k$-раз дифференцируемое отображение, то это равенство определяет непрерывный линейный функционал на $H^{k}$.

Заметим, что, если обобщенная функция $g \in H^{-k}$ имеет вид $g=$ $g(x) d x$, то $g \circ \psi_{t, s}$ — это композиция плотности $g$ и отображения $\psi_{t, s}$

$$
\begin{aligned}
\left\langle g \circ \psi_{s, t}, h\right\rangle & =\int_{\mathbf{R}^{d}} g\left(\psi_{s, t}(x)\right) h(x) d x \\
& =\int_{\mathbf{R}^{d}} g(y) h\left(\phi_{s, t}(y)\right) J_{s, t}\left(\phi_{s, t}(y)\right) d y \quad \forall h \in \mathscr{D},
\end{aligned}
$$

что вытекает из формулы замены переменных под знаком интеграла.

Наша цель - построить вероятностное представление решения задачи $(1.1),(1.2)$, исходя из предположения о том, что оператор $\mathscr{L}_{u}^{q}$ вида (1.5) определен в обобщенном смысле, т.е. в смысле интегрального тождества

$$
\left\langle\mathscr{L}_{u}^{q} g, h\right\rangle=\left\langle g, \mathscr{M}_{u}^{q} h\right\rangle,
$$

где $\mathscr{M}_{u}^{q}$ - соответствующий дифференциальный оператор. При этом мы рассмотрим два варианта системы (1.1), (1.2) как при отсутствии кросс-диффузионных членов, так и при их наличии.

Пусть $v(t, x) \in R^{2}$ - заданная ограниченная функция, дифференцируемая по пространственной переменной. Для того, чтобы построить соответствующие вероятностные представления, нам понадобится ряд вспомогательных результатов, получение которых можно существенно упростить, если рассмотреть СДУ (2.12) в форме Стратоновича. Напомним, что стохастические дифференциалы в форме Ито $d \xi(t)=M_{v}^{q}(x i(t)) d w(t)$ и в форме Стратоновича $d^{S} \xi(t)=M_{v}^{q}(\xi(t)) \circ d w(t)$ связаны соотношением

$$
\begin{aligned}
d^{S} \xi(t) & =M_{v}^{q}(\xi(t)) \circ d w(t) \\
& =\frac{1}{2} \beta_{v}^{q}(\xi(t)) d t+M_{v}^{q}(\xi(t)) d w(t)=d \xi(t)+\frac{1}{2} \beta_{v}^{q}(\xi(t)) d t
\end{aligned}
$$

где

$$
\beta_{v}^{q}(x)=M_{v}^{q}(x) \nabla M_{v}^{q}(x)=\frac{\alpha_{q q} \nabla v^{q}(x)}{2}
$$


Перепишем уравнение (2.12) в виде

$$
d \xi^{q}(\theta)=-\frac{1}{2} \beta_{v}^{q}\left(\xi^{q}(\theta)\right) d \theta-M_{v}^{q}\left(\xi^{q}(\theta)\right) \circ d w(\theta), \quad \xi^{q}(s)=y .
$$

Из теоремы 4.2.2 [10] вытекает следующее утверждение.

Теорема 2.1. Пусть $\phi_{s, t}^{q}(y)=\xi^{q}(t)$ удовлетворяет СДУ $(2.14)$, коэффициент диффузии которого принадлежат классу $C^{k+1} n p u(k \geqslant 1)$. Тогда обратный поток $\left[\phi_{s, t}^{q}\right]^{-1}=\psi_{s, t}^{q}$ удовлетворяет СДУ

$$
d \psi_{t, x}^{q}(\theta)=-\widehat{J}_{\theta, t}^{q}\left(\psi_{t, x}(\theta)\right) \beta_{v}^{q}(x) d \theta+\widehat{J}_{\theta, t}^{q}\left(\psi_{t, x}(\theta)\right) M_{v}^{q}(x) \circ d w(\theta) .
$$

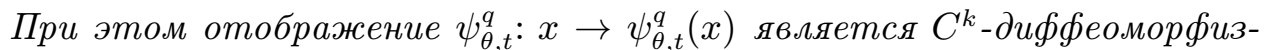
мом.

Сужение производящего оператора $\mathscr{M}^{q}$ эволюционного семейства

$$
U^{q}(s, t) h(y)=\mathbf{E}\left[h\left(\xi_{s, y}^{q}(t)\right)\right]
$$

на соболевское пространство $H^{2}$ можно вычислить с использованием классической формулы Ито. Производящий оператор $\mathscr{L}_{v}^{q}=\left[\mathscr{M}_{v}^{q}\right]^{+}$двойственной полугруппы $\left[U^{q}\right]^{+}(t, s)$ - это двойственный оператор по отношению к $\mathscr{M}$ в смысле спаривания соболевских пространств $H^{k}$ и $H^{-k}$ ( а также в смысле скалярного произведения в $L^{2}\left(\mathbf{R}^{d}\right)$ или $H^{2}$ ) . Для вычисления генератора эволюционного семейства $\left[U^{q}\right]^{+}(t, s)$, используя его вероятностное представление, воспользуемся обобщенной формулой Ито (см. [12]).

Лемма 2.1 (Обобщенная формула Ито). Пусть $g(t)$ - непрерывная функиия со значениями в $H^{k}$ для некоторого $k \in\{-2,-1,0,1,2\}$, стохастический поток $\phi_{s, t}^{q}$ задан соотношением вида (2.1) u $\psi_{t, s}^{q}$ это соответствующий обращенный по времени стохастический поток. Тогда справедливы соотношения

$$
\begin{aligned}
g(t) \circ \phi_{s, t}^{q}= & g(s)+\int_{s}^{t} \frac{\partial g(\theta)}{\partial \theta} \circ \phi_{s, \theta}^{q} d \theta+\int_{s}^{t} \nabla g(\theta) \circ \phi_{s, \theta}^{q} \cdot d \phi_{s, \theta}^{q} \\
& +\int_{s}^{t}\left[\mathscr{N}_{v}^{q} g(\theta)\right] \circ \phi_{s, \theta} d \theta
\end{aligned}
$$

$u$

$$
\begin{aligned}
g(t) \circ \psi_{s, t}^{q}= & g(t)+\int_{s}^{t} \frac{\partial g(\theta)}{\partial \theta} \circ \psi_{s, \theta}^{q} d \theta-\int_{s}^{t} \nabla g(\theta) \circ \psi_{s, \theta}^{q} \cdot d \psi_{s, \theta}^{q} \\
& +\int_{s}^{t} \mathscr{N}_{v}^{q}\left[g(t) \circ \psi_{s, \theta}\right] d \theta
\end{aligned}
$$

¿de

$$
\mathscr{N}_{v}^{q} g=\frac{1}{2}\left(\alpha_{q}+\alpha_{q q} v^{q}\right) \Delta g+\frac{1}{4} \nabla\left[\alpha_{q q} v^{q}\right] \nabla g .
$$

При этом для обобщенной функчии $g$ действие операторов $\mathscr{N}_{u}^{q}$ понимается в смисле теории обобщенных функций. 


\section{Рассмотрим систему СДУ}

$$
\begin{gathered}
\left.d \widehat{\xi}_{0, x}^{q}(\theta)=\widehat{J}_{\theta, 0}^{q}\left(\widehat{\xi}_{0, x}^{q}(\theta)\right) \beta_{v}^{q}(x)\right) d \theta+\widehat{J}_{\theta, 0}^{q}\left(\widehat{\xi}_{0, x}^{q}(\theta)\right) M_{v}^{q}(x) \circ d w(\theta), \\
d \widehat{J}^{q}(\theta)=\widehat{J}^{q}(\theta) \nabla M_{v}^{q}\left(\widehat{\xi}_{0, x}^{q}(\theta)\right) \circ d w(\theta)+\widehat{J}^{q}(\theta)\left[\nabla \beta_{v}^{q}\left(\widehat{\xi}_{0, x}^{q}(\theta)\right)\right]^{2} d \theta, \quad \widehat{J}^{q}(0)=I .
\end{gathered}
$$

Обозначим $\mathscr{L}_{v}^{q}$ оператор, формально сопряженный к оператору

$$
\mathscr{M}_{v}^{q}=\frac{1}{2}\left(\alpha_{q}+\alpha_{q q} v^{q}\right) \Delta, \quad \mathscr{L}_{v}^{q}=\left[\mathscr{M}_{v}^{q}\right]^{+} .
$$

Используя формулу интегрирования по частям, нетрудно проверить, что оператор $\mathscr{L}_{v}^{q}$ действует по правилу

$$
\begin{aligned}
\int_{\mathbf{R}^{d}} \mathscr{L}_{v}^{q} g^{q}(x) h(x) d x & =\frac{1}{2} \int_{\mathbf{R}^{d}} g^{q}(x)\left(\alpha_{q}+\alpha_{q q} v^{q}\right) \Delta h d x \\
& =\frac{1}{2} \int_{\mathbf{R}^{d}} \Delta\left[g^{q}\left(\alpha_{q}+\alpha_{q q} v^{q}\right)\right] h(x) d x .
\end{aligned}
$$

Заметим, что, при сделанных выше априорных предположениях, мы находимся в рамках теории Куниты и можем сформулировать следующее предложение (вытекающее из теоремы 2.1 работы [12]).

Теорема 2.2. Пусть $u_{0}^{q} \in H^{k}\left(\mathbf{R}^{d}\right)$ - заданная функиия, $u^{q}(t)-$ ограниченные обобщенные решения задачи (1.1), (1.2) из класса $H^{k}\left(\mathbf{R}^{d}\right)$, $\alpha_{12}=\alpha_{21}=0 u$

$$
\kappa^{q}(t)=\exp \left(\int_{0}^{t} m_{u}^{q} \circ \phi_{0, \theta} d \theta\right) u_{0}^{q} .
$$

Тогда справедливо представление $u^{q}(t)=\mathbf{E}\left[\kappa^{q}(t) \circ \psi_{0, t}^{q}\right], q=1,2$, где $\psi_{0, t}^{q}$ - стохастический поток порожденный решением СДУ (2.23) при $u \equiv v$.

При этом существует положительная константа $C$, зависящая только от $k, T$ и $\mathscr{L}^{q}$, для которой справедлива оченка

$$
\left\|u^{q}(t)\right\|_{k}^{2} \leqslant C^{q}\left\|u_{0}\right\|_{k}^{2}
$$

Д о к а з а т е л ь с т в о. Если $u_{0}^{q} \in H^{2}$, то $\kappa^{q}(t)-$ непрерывный семимартингал. Поскольку $\kappa^{q}(t)$ удовлетворяет ОДУ

$$
\frac{d \kappa^{q}(t)}{d t}=m_{u}^{q} \kappa^{q}(t), \quad \kappa^{q}(0)=u_{0}^{q}
$$

то из обобщенной формулы Ито следует, что

$$
\begin{aligned}
\kappa^{q}(t) \circ \psi_{0, t}^{q}= & u_{0}^{q}+\int_{0}^{t} \frac{\partial \kappa^{q}(\theta)}{\partial \theta} \circ \psi_{\theta, t}^{q} d \theta+\int_{0}^{t} \nabla\left(\kappa^{q}(\theta) \circ \psi_{\theta, t}^{q}\right) \cdot d \psi_{\theta, t}^{q} \\
& +\int_{0}^{t} \mathscr{L}_{u}^{q}\left(\kappa^{q}(\theta) \circ \psi_{\theta, t}^{q}\right) d \theta
\end{aligned}
$$


или, с учетом (2.26),

$\kappa^{q}(t) \circ \psi_{0, t}^{q}=u_{0}^{q}+\int_{0}^{t} \nabla\left(\kappa^{q}(t) \circ \psi_{\theta, t}^{q}\right) \cdot M_{u}^{q}(x) d w(\theta)+\int_{0}^{t} \mathscr{L}_{u}^{q}\left(\kappa^{q}(\theta) \circ \psi_{\theta, t}^{q}\right) d \theta$,

где $\mathscr{L}_{u}^{q}(x)$ - оператор, сопряженный к оператору

$$
\mathscr{M}_{u}^{q}(x)=\frac{1}{2}\left[M_{u}^{q}(x)\right]^{2} \Delta+m_{u}^{q}(x),
$$

в смысле интегрального тождества

$$
\left\langle\mathscr{L}_{u}^{q} g, h\right\rangle=\int_{\mathbf{R}^{d}} \mathscr{L}_{u}^{q}(x) g(x) h(x) d x=\int_{\mathbf{R}^{d}} g(x) \mathscr{M}_{u}^{q}(x) h(x) d x=\left\langle g, \mathscr{M}_{u}^{q} h\right\rangle,
$$

справедливого для любой тестовой функции $h$.

Вычислим математическое ожидание обеих частей соотношения (2.28). Поскольку

$$
\mathbf{E}\left\langle\int_{0}^{t} \nabla\left(\kappa^{q}(t) \circ \psi_{t, \theta}^{q}\right) \cdot M_{u}^{q}(x) d w(\theta), h\right\rangle=0,
$$

И

$$
\begin{aligned}
\mathbf{E}\left[\left\langle\int_{0}^{t}\left[\mathscr{L}_{u}^{q}\right]\left(\kappa^{q}(\theta) \circ \psi_{\theta, 0}^{q}\right) d \theta, h\right\rangle\right] & =\mathbf{E}\left[\int_{0}^{t}\left\langle\kappa^{q}(\theta) \circ \psi_{\theta, t}^{q}, \mathscr{M}_{u}^{q} h\right\rangle d \theta\right] \\
=\int_{0}^{t}\left\langle\mathbf{E}\left[\kappa^{q}(\theta) \circ \psi_{\theta, t}^{q}\right], \mathscr{M}_{u}^{q} h\right\rangle d \theta & =\int_{0}^{t}\left\langle\left[\mathscr{L}_{u}^{q}\right] \mathbf{E}\left[\kappa^{q}(\theta) \circ \psi_{\theta, t}^{q}\right], h\right\rangle d \theta,
\end{aligned}
$$

то получим равенство

$$
\mathbf{E}\left[\kappa^{q}(t) \circ \psi_{0, t}^{q}\right]=u_{0}^{q}+\int_{0}^{t}\left\langle\mathscr{L}_{u}^{q} \mathbf{E}\left[\kappa^{q}(\theta) \circ \psi_{\theta, t}^{q}\right], h\right\rangle d \theta .
$$

Вычисляя производную по переменной $t$ от обеих частей соотношения $(2.30)$, получим, что функции $g^{q}(t)=\mathbf{E}\left[\kappa^{q}(t) \circ \psi_{0, t}^{q}\right]$ удовлетворяют задаче Коши (1.1), (1.2) при $\alpha_{12}=\alpha_{21}=0$, и, поскольку $\lim _{t \rightarrow 0}\left\langle g^{q}(t), h\right\rangle=$ $\left\langle u_{0}^{q}, h\right\rangle$, то $\lim _{t \rightarrow 0} g^{q}(t)=u_{0}^{q}$.

Покажем, что $g^{q}(t) \equiv u^{q}(t)$. Предположим, что существует два обобщенных решения $\left(g^{1}, g^{2}\right)$ и $\left(u^{1}, u^{2}\right)$ рассматриваемой задачи. Тогда их разность $G^{q}(t)=g^{q}(t)-u^{q}(t)$ удовлетворяет задаче Коши

$$
\frac{\partial G^{q}(t)}{\partial t}=\mathscr{L}_{u}^{q}(t) G^{q}(t), \quad G^{q}(0)=0
$$

Покажем, что при этом $G^{q}(t)=0$. Пусть $T>0$ и $h \in C_{0}^{2} \cap H^{2}$. Тогда существует решение $h^{q}(s, y)$ задачи Коши

$$
h_{s}^{q}(s, y)+\mathscr{M}_{u}^{q} h^{q}(s, y)=0, \quad h^{q}(T, y)=h(y),
$$


и при этом $h^{q}(s)$ принадлежит $C^{2}\left(\mathbf{R}^{d}\right)$. Отсюда вытекает, что для любой функции $h \in C_{0}^{2} \cap H^{2}$ справедливы соотношения

$$
\begin{aligned}
\left\langle G^{q}(t), h(t)\right\rangle & =\int_{0}^{t}\left\langle\frac{d}{d \theta} G^{q}(\theta), h(\theta)\right\rangle d \theta+\int_{0}^{t}\left\langle G^{q}(\theta), \frac{\partial h(\theta)}{\partial \theta}\right\rangle d \theta \\
& =\int_{0}^{t}\left\langle\mathscr{L}_{u}^{q} G^{q}(\theta), h(\theta)\right\rangle d \theta-\int_{0}^{t}\left\langle G^{q}(\theta), \mathscr{M}_{u}^{q} h(\theta)\right\rangle d \theta=0,
\end{aligned}
$$

следовательно, $G^{q}(t)=0$. Последнее утверждение теоремы вытекает из следующего утверждения ([11], теорема 2.1). Теорема доказана.

Лемма 2.2. Пусть $g(t), g_{0} \in H^{k} \cap C\left(\mathbf{R}^{d}\right)$ u $\widehat{\xi}(t)=\psi_{s, t}^{q}(x)$ удовлетворяют СДУ (2.23). Тогда существует $t_{0}>0$ и константа $C_{k, t_{0}}^{q}$, для которой справедливо неравенство

$$
\mathbf{E}\left[\left\|g_{0} \circ \psi_{s, t}^{q}\right\|_{k}^{2}\right] \leqslant C_{k, t_{0}}^{q}\left\|g_{0}\right\|_{k}^{2} \quad \forall s, t \in\left[0, t_{0}\right] .
$$

При этом, если случайная величина $G$ не зависит от потока $\psi_{s, t}^{q}$ то справедлива оченка

$$
\mathbf{E}\left[\left\|G \circ \psi_{s, t}^{q}\right\|_{k}^{2}\right] \leqslant C_{k, t_{0}}^{q} \mathbf{E}\left[\|G\|_{k}^{2}\right] \quad \forall s, t \in\left[0, t_{0}\right] .
$$

3. СДУ, ассоциированные с системой параболических уравнений без кросс диффузии. Анализ результатов, полученных в предыдущих параграфах, показывает, что решение задачи Коши (1.1), (1.2) при $\alpha_{12}=\alpha_{21}=0$ можно свести к исследованию следующей системы стохастических соотношений

$$
\begin{gathered}
d \xi^{q}(\theta)=-\frac{1}{2} \beta_{u^{1}, u^{2}}^{q}\left(\xi^{q}(\theta)\right) d \theta-M_{u}^{q}(\xi(\theta)) \circ d w(\theta), \quad \xi^{q}(0)=y, \\
d \widehat{\xi}_{0, x}^{q}(\theta)=-\frac{1}{2} \widehat{J}_{\theta, 0}^{q}\left(\widehat{\xi}_{0, x}^{q}(\theta)\right) \beta_{u^{1}, u^{2}}^{q}(x) d \theta+\widehat{J}_{\theta, 0}^{q}\left(\widehat{\xi}_{0, x}^{q}(\theta)\right) M_{u}^{q}(x) \circ d w(\theta), \quad(3.2) \\
d \widehat{J}^{q}(\theta)=\widehat{J}^{q}(\theta) \nabla M_{u}^{q}\left(\widehat{\xi}_{0, x}^{q}(\theta)\right) \cdot d w(\theta)+\widehat{J}^{q}(\theta)\left[\nabla M_{u}^{q}\left(\widehat{\xi}_{0, x}^{q}(\theta)\right)\right]^{2} d \theta, \quad \widehat{J}^{q}(0)=I . \\
\left.u^{q}(t)=\mathbf{E}\left(\exp \left(\int_{0}^{t} m_{u}^{q} \circ \psi_{\theta, t}^{q}\right) d \theta\right) u_{0}^{q} \circ \psi_{0, t}^{q}\right) .
\end{gathered}
$$

Заметим, что, наряду с уравнением (3.2) для обращенного по времени процесса $\widehat{\xi}^{q}(t)$, можно воспользоваться также уравнением $(2.15)$ и рассмотреть следующие интегральные стохастические уравнения

$$
\begin{aligned}
\widehat{\xi}^{q}(\tau)=\xi^{q}(t)+ & \int_{0}^{\tau}\left(M_{u}^{q} \nabla M_{u}^{q}\right)\left(\widehat{\xi}^{q}(\theta)\right) d \theta+\int_{0}^{\tau} M_{u}^{q}\left(\widehat{\xi}^{q}(\theta)\right) d \widetilde{w}(\theta), \\
\widehat{J}^{q}(\tau)= & I+\int_{\tau}^{t} \widehat{J}^{q}(\theta) \nabla M_{u}^{q}\left(\widehat{\xi}_{0, x}^{q}(\theta)\right) \cdot d w(\theta) \\
& +\int_{\theta}^{t} \widehat{J}^{q}(\theta)\left[\nabla M_{u}^{q}\left(\widehat{\xi}_{0, x}^{q}(\theta)\right)\right]^{2} d \theta, \quad \widehat{J}^{q}(0)=I .
\end{aligned}
$$


Поскольку коэффициенты сноса в уравнениях (3.5), (3.6) зависят от $\nabla u^{q}$, то для того, чтобы получить замкнутую систему соотношений, нужно систему (3.4)-(3.6) пополнить вероятностным представлением для вектор-функции $\nabla u^{q}$. Для этого нужно продифференцировать исходную задачу и построить вероятностное представление для ее дифференциального продолжения (см. [9]). Формальное дифференцирование соотношения (3.4) позволяет получить соотношение

$$
\begin{aligned}
\nabla u^{q}(t)= & \mathbf{E}\left[\int_{0}^{t} \nabla m_{u}^{q}\left(\psi_{0, \theta}^{q}\right) \widehat{\mathbf{J}}^{q}(\theta) d \theta \exp \left(\int_{0}^{t} m_{u^{1}, u^{2}}^{q}\left(\psi_{\theta, t}^{q}\right) d \theta\right) u_{0}^{q} \circ \psi_{0, t}^{q}\right] \\
& +\mathbf{E}\left[\exp \left(\int_{0}^{t} m_{u}^{q}\left(\psi_{\theta, t}^{q}\right) d \theta\right) \nabla u_{0}^{q} \circ \psi_{0, t}^{q} \widehat{\mathbf{J}}^{q}(t)\right] .
\end{aligned}
$$

Мы вернемся к рассмотрению этой системы стохастических уравнений ниже, а пока рассмотрим стохастическую систему, ассоциированную с задачей (1.1), (1.2), предполагая, что $\alpha_{12}=\alpha_{21}=a_{q}=b_{q}=c_{q}=0$. При этом уравнения (3.5)-(3.6) сохраняются, а функции $u^{q}(t), \nabla u^{q}(t)$ задаются соотношениями

$$
\begin{gathered}
u^{q}(t)=\mathbf{E}\left[u_{0}^{q} \circ \psi_{0, t}^{q}\right], \\
\nabla u^{q}(t)=\mathbf{E}\left[\nabla u_{0}^{q} \circ \psi_{0, t}^{q} \widehat{\mathbf{J}}^{q}(t)\right] .
\end{gathered}
$$

Для того, чтобы построить решение системы (3.5), (3.6), (3.8), (3.9), рассмотрим семейство последовательных приближений вида

$$
\begin{gathered}
\widehat{\xi}_{n}^{q}(\tau)=x+\int_{0}^{\tau} \beta_{u_{n}}^{q}\left(\widehat{\xi}_{n}^{q}(\theta)\right) d \theta+\int_{0}^{\tau} M_{u_{n}}^{q}\left(\widehat{\xi}_{n}^{q}(\theta)\right) d \widetilde{w}(\theta) \\
\widehat{J}_{n}^{q}(\tau)=I+\int_{\tau}^{t} \widehat{J}_{n}^{q}(\theta) \nabla M_{u_{n}}^{q}\left(\widehat{\xi}^{q}(\theta)\right) \cdot d w(\theta)+\int_{\tau}^{t} \widehat{J}_{n}^{q}(\theta)\left[\nabla M_{u_{n}}^{q}\left(\widehat{\xi}_{n}^{q}(\theta)\right)\right]^{2} d \theta \\
u_{n+1}^{q}(t)=\mathbf{E}\left[u_{0}^{q}\left(\widehat{\xi}_{n}^{q}(t)\right)\right] \\
\nabla u_{n+1}^{q}(t)=\mathbf{E}\left[\nabla u_{0}^{q}\left(\widehat{\xi}_{n}^{q}(t)\right) \widehat{\mathbf{J}}_{n}^{q}(t)\right]
\end{gathered}
$$

Наша цель - доказать сходимость построенной системы последовательных приближений и изучить свойства предельных функций $u^{q}(t)$. Для достижения этой цели нам потребуется ряд вспомогательных утверждений.

Пусть $v^{q}, q=1,2$ - заданные функции. Будем говорить, что для функции $v^{q}$ выполнены условия С 3.1, если $v^{q} \in \mathscr{W}^{1}, \inf _{x} v^{q}(t, x) \geqslant 0$. $\left(v^{1}, v^{2}\right)$.

Обозначим $K_{v^{q}}(t)=\sup _{x}\left[v^{q}(t, x)\right]^{2}, K_{v^{q}}^{1}(t)=\sup _{x}\left\|\nabla v^{q}(t, x)\right\|^{2}$ и $v=$

Рассмотрим вспомогательные уравнения вида

$$
\widehat{\xi}_{v^{q}}^{q}(\tau)=x+\int_{0}^{\tau} \beta_{v^{q}}^{q}\left(\widehat{\xi}_{v^{q}}^{q}(\theta)\right) d \theta+\int_{0}^{\tau} M_{v^{q}}^{q}\left(\widehat{\xi}_{v^{q}}^{q}(\theta)\right) d \widetilde{w}(\theta),
$$




$$
\begin{gathered}
\widehat{J}_{v}^{q}(\tau)=I+\int_{0}^{\tau} \widehat{J}_{v^{q}}^{q}(\theta) \nabla M_{v^{q}}^{q}\left(\widehat{\xi}^{q}(\theta)\right) \cdot d w(\theta)+\int_{0}^{\tau} \widehat{J}_{v^{q}}^{q}(\theta)\left[\nabla M_{v^{q}}^{q}\left(\widehat{\xi}_{v^{q}}^{q}(\theta)\right)\right]^{2} d \theta, \\
u_{v^{q}}^{q}(t)=\mathbf{E}\left[u_{0}^{q} \circ \psi_{0, t}^{q}\right],
\end{gathered}
$$

где $\psi_{0, t}^{q}(x)=\widehat{\xi}_{v^{q}}^{q}(t)$,

$$
\nabla u_{v^{q}}^{q}(t)=\mathbf{E}\left[\nabla u_{0}^{q} \circ \psi_{0, t}^{q} \widehat{\mathbf{J}}_{v^{q}}^{q}(t)\right]
$$

Напомним, что

$$
\beta_{v}^{q}(x)=M_{v^{q}}^{q}(x) \nabla M_{v^{q}}^{q}(x)=\frac{1}{2}\left[\alpha_{q q} \nabla v^{q}(x)\right],
$$

обозначим $\sup _{x}\|u(x)\|=\|u\|_{\infty}$ и пусть $\|x\|$ обозначает норму элемента $x$ в соответствующем пространстве, в частности, если $J^{q}$ - матрица, то $\left\|J^{q}\right\|$ обозначает матричную норму.

Лемма 3.1. Пусть $v^{q} \in \mathscr{W}^{1}$ удовлетворяют условию С 3.1. Тогда существуют процессьл $\widehat{\xi}_{v}^{q}(\theta), \widehat{\mathbf{J}}_{v}^{q}(\theta)$, удовлетворяюшие уравнениям (3.14), (3.15) соответственно, и справедливь оченки

$$
\mathbf{E}\left\|\widehat{\xi}^{q}(\theta)\right\|^{2} \leqslant 3\left[\|x\|^{2}+\frac{\theta}{4} \int_{0}^{\theta} \alpha_{q q}^{2} K_{v^{q}}^{1}(\tau) d \tau+\int_{0}^{\theta}\left[\alpha_{q}+\alpha_{q q} K_{v}(\tau)\right] d \tau\right],
$$

при $0 \leqslant \theta \leqslant T$, где константа $T$ будет определена ниже, $и$

$$
\mathbf{E}\left\|\widehat{\mathbf{J}}_{v^{q}}^{q}(\theta)\right\|^{2} \leqslant \exp \left(\int_{0}^{\theta} \Lambda_{q} K_{v^{q}}^{1}(\tau) d \tau\right)
$$

где $\Lambda_{q}=\left(5 \alpha_{q q}^{2}\right) /\left(4 \alpha_{q}\right)$.

Д о к а з а т е л ь с т в о. Воспользовавшись стандартными оценками и условием $\mathbf{C ~ 3 . 1 , ~ н е т р у д н о ~ п р о в е р и т ь , ~ ч т о ~}$

$$
\mathbf{E} \| \widehat{\xi}_{v^{q}}^{q}\left(\theta \|^{2} \leqslant 3\left[\|x\|^{2}+\frac{\theta}{4} \int_{0}^{\theta} \alpha_{q q}^{2} K_{v^{q}}^{1}(\tau) d \tau+\int_{0}^{\theta}\left[\alpha_{q}+\alpha_{q q} K_{v^{q}}(\tau)\right] d \tau\right] .\right.
$$

Применяя формулу Ито к оценке решения линейного СДУ (3.15), получим неравенство

$$
\mathbf{E}\left\|\widehat{\mathbf{J}}_{v^{q}}^{q}(\theta)\right\|^{2} \leqslant I+\mathbf{E}\left[\frac{5}{4} \int_{0}^{\theta} \frac{\alpha_{q q}^{2}\left\|\nabla v^{q}\right\|^{2}\left(\tau, \widehat{\xi}^{q}(\tau)\right)}{\left[\alpha_{q}+\alpha_{q q} v^{q}\left(\tau, \xi_{v^{q}}^{q}(\tau)\right)\right]}\left\|\widehat{\mathbf{J}}_{v^{q}}^{q}(\tau)\right\|^{2} d \tau\right]
$$

из которого, в силу леммы Гронуолла, вытекает оценка

$$
\mathbf{E}\left\|\widehat{\mathbf{J}}_{v^{q}}^{q}(\theta)\right\|^{2} \leqslant \exp \left(\int_{0}^{\theta} \Lambda_{q} K_{v^{q}}^{1}(\tau) d \tau\right)
$$

где $\Lambda_{q}=\left(5 \alpha_{q q}^{2}\right) /\left(4 \alpha_{q}\right)$. Лемма доказана. 
Лемма 3.2. Пусть вьгполнень условия леммы 3.1 и $u_{0 q} \in H^{2}-$ ограниченные функчии, подчиняющиеся оценке inf $u_{0 q}(x) \geqslant 0$. Тогда для некоторой положительной константь $T u 0 \leqslant t \leqslant T$ справедливь оченки

$$
\begin{gathered}
\left\|u_{v^{q}}^{q}(t)\right\|_{\infty}^{2} \leqslant K_{0 q} \\
\left\|\nabla u_{v^{q}}^{q}(t)\right\|_{\infty}^{2}=K_{u^{q}}^{1}(t) \leqslant K_{0 q}^{1} \exp \left(\int_{0}^{t} \Lambda_{q} K_{v^{q}}^{1}(\tau) d \tau\right) .
\end{gathered}
$$

Д о к а з а т е л ь с т в о. Первая оценка непосредственно вытекает из формулы (3.16) с учетом условий на $u_{0 q}$ и $v^{q}$. Для доказательства второй оценки воспользовавшись формулой (3.17) и определением функций $K_{v}(\theta), K_{v}^{1}(\theta)$, получим

$$
\left\|\nabla u_{v^{q}}^{q}(t)\right\|_{\infty}^{2} \leqslant K_{0 q}^{1} E\left\|\widehat{\mathbf{J}}^{q}(t)\right\|^{2}
$$

откуда с учетом (3.18) следует

$$
\left.\left\|\nabla u^{q}(t)\right\|_{\infty}^{2} \leqslant K_{0 q}^{1} \exp \left(\int_{0}^{t} \Lambda_{q} K_{v^{q}}^{1}(\tau)\right] d \tau\right), \quad 0 \leqslant t \leqslant T
$$

где $T$ - некоторая константа, которая будет выбрана ниже. Из полученных оценок вытекает, что функции $K_{u^{q}}^{1}(t)=\left\|\nabla u_{q}^{1}(t)\right\|_{\infty}^{2}$ удовлетворяют оценкам

$$
K_{u^{q}}^{1}(t) \leqslant K_{0}^{1} \exp \left(\int_{0}^{t} \Lambda_{q} K_{v^{q}}^{1}(\tau) d \tau\right)
$$

Лемма доказана.

В качестве завершающего предварительного результата сформулируем следующее утверждение.

Лемма 3.3. Пусть выполнены условия леммы 3.2 и справедливь оценки $\left\|K_{v^{q}}(\theta)\right\| \leqslant \kappa^{q}(\theta), K_{v^{q}}^{1}(\theta) \leqslant \kappa_{1}^{q}(\theta)$ для положительньх функций $\kappa^{q}(\theta), \kappa_{1}^{q}(\theta)$, ограниченньх на интервале $[0, T]$ для некоторого $T>0$. Тогда существует интервал $\left[0, T_{1}\right], 0<T_{1} \leqslant T$, такой, что функиии $u^{q}(\theta), \nabla u^{q}(\theta)$ вида (3.15), (3.16) подчиняются оченкам

$$
K_{u^{q}}(\theta) \leqslant \kappa^{q}(\theta), \quad K_{u^{q}}^{1}(\theta) \leqslant \kappa_{1}^{q}(\theta)
$$

$\partial л я$ всех $\theta \in\left[0, T_{1}\right]$.

Д ок а з а т е ль с т в о. Из оценок (3.18)-(3.20) леммы 3.1 и леммы 3.2 следует, что справедливы неравенства $K_{u^{q}}(t) \leqslant K_{0 q}$ откуда следует, что в качестве $\kappa^{q}(t)$ можно выбрать $\kappa^{q}(t)=K_{0 q}$.

Для доказательства второй оценки рассмотрим вспомогательное уравнение

$$
X(t)=K_{0 q}^{1} \exp \left\{\int_{0}^{t}\left[\Lambda_{q} X(\tau)\right] d \tau\right\}
$$


и заметим, что функция $X(t)$, удовлетворяющая интегральному уравнению (3.23), удовлетворяет также задаче Коши

$$
\frac{d X(t)}{d t}=\left[\Lambda_{q} X(t)\right] X(t), \quad X(0)=K_{0 q}^{1}
$$

При этом решение задачи Коши (3.24) имеет вид

$$
X(t)=\frac{K_{0}^{1}}{1-\Lambda_{q} K_{0 q}^{1} t}
$$

и ограничено на интервале $[0, T]$, длина которого $T$ подчиняется оценке

$$
T<\min \left(\frac{1}{\Lambda_{1} K_{01}^{1}}, \frac{1}{\Lambda_{2} K_{02}^{1}}\right) .
$$

Наконец, из соотношений $(3.25),(3.26)$ следует, что в качестве $\kappa_{1}^{q}(t)$ можно выбрать $\kappa_{1}^{q}(t)=K_{0 q}^{1} /\left(1-\Lambda_{q} K_{0 q}^{1} t\right)$ для всех $t<T$. Лемма доказана.

Применяя полученные в леммах 3.1-3.3 оценки к системе последовательных приближений, покажем, что процессы $\widehat{\xi}_{n}^{q}(t), \widehat{\mathbf{J}}_{n}^{q}(t)$ и функции $u_{n}^{q}(t), \nabla u_{n}^{q}(t)$, удовлетворяющие (3.10)-(3.13), сходятся к решению системы (3.4)-(3.7).

Теорема 3.1. Пусть выполнень условия леммь 2.3. Тогда проиессьл $\widehat{\xi}_{n}^{q}(t), \widehat{\mathbf{J}}_{n}^{q}(t)$ и функиии $u_{n}^{q}(t), \nabla u_{n}^{q}(t)$, удовлетворяющие (3.10)(3.13), сходятся в соответствующих нормах $\kappa$ решению системь (3.4)(3.7).

Д ок а з а т е л ь с т в о. Обозначим $C=2 K_{0}^{1}(T+1), M_{n}^{q}=M_{u^{n}}^{q}$, $\beta_{n}^{q}=\beta_{u^{n}}^{q}$ и оценим разность

$$
\begin{aligned}
\left|u_{n+1}^{q}(t, x)-u_{n}^{q}(t, x)\right|^{2}= & \mathbf{E}\left|u_{0}^{q}\left(\xi_{n}^{q}(t)\right)-u_{0}^{q}\left(\xi_{n-1}^{q}(t)\right)\right|^{2} \\
\leqslant & K_{0}^{1} \mathbf{E}\left\|\xi_{n}^{q}(t)-\xi_{n-1}^{q}(t)\right\|^{2} \\
\leqslant & C\left[\int_{0}^{t} \mathbf{E}\left\|\beta_{n}^{q}\left(\widehat{\xi}_{n}^{q}(\theta)\right)-\beta_{n-1}^{q}\left(\widehat{\xi}_{n-1}^{q}(\theta)\right)\right\|^{2} d \theta\right. \\
& \left.\quad+\left.\int_{0}^{t} \mathbf{E}\left\|M_{n}^{q}\left(\widehat{\xi}_{n}^{q}(\theta)\right)-M_{n-1}^{q}\left(\widehat{\xi}_{n-1}^{q}(\theta)\right)\right\|^{2} d \theta\right|^{2}\right] \\
\leqslant & C \int_{0}^{t} \int_{0}^{\theta} K^{1}(\tau) \mathbf{E}\left\|\xi_{n}^{q}(\tau)-\xi_{n-1}^{q}(\tau)\right\|^{2} d \tau d \theta \\
& \quad+C \mathbf{E} \int_{0}^{t}\left|u_{n}^{q}\left(\theta, \widehat{\xi}_{n}^{q}(\theta)\right)-u_{n-1}^{q}\left(\theta, \widehat{\xi}_{n}^{q}(\theta)\right)\right|^{2} d \theta \\
\leqslant & \cdots \leqslant C^{n} \int_{0}^{t} \cdots \int_{0}^{t_{n}}\left[\mathbf{E}\left\|\widehat{\xi}_{1}^{q}(\tau)-x\right\|^{2}\right. \\
& \left.+\mathbf{E}\left|u_{1}^{q}\left(\tau, \widehat{\xi}_{1}^{q}(\tau)\right)-u_{0}^{q}\left(\widehat{\xi}_{1}^{q}(\tau)\right)\right|^{2}\right] d \tau d t_{n} \cdots d t_{1} \\
\leqslant & \frac{C^{n} T^{n}}{n !} G
\end{aligned}
$$


где константа $G$ зависит только от $K_{0} K_{0}^{1}$. Из полученной оценки следует, что

$$
\lim _{n \rightarrow \infty} \sup _{x}\left|u_{n+1}^{q}(t, x)-u_{n}^{q}(t, x)\right|=0
$$

и аналогично можно показать, что для любых $n, m \rightarrow \infty$

$$
\lim _{n, m \rightarrow \infty} \sup _{x}\left|u_{n}^{q}(t, x)-u_{m}^{q}(t, x)\right|=0
$$

В силу полноты пространства непрерывных функций существует предел

$$
u^{q}(t)=\lim _{n \rightarrow \infty} u_{n}(t)
$$

при каждом $t \in\left[0, T_{1}\right], T_{1}<T$ и, в силу леммы 3.3 , предельная функция $u^{q}(t, x)$ обладает ограниченной производной по пространственной переменной. Наконец, из полученных оценок нетрудно вывести, что предельная функция $u(t)=\left(u^{1}(t), u^{2}(t)\right)$ ограничена на интервале $\left[0, T_{1}\right]$ и справедливо соотношение

$$
\lim _{n, m \rightarrow \infty} \sup _{t \in\left[0, T_{1}\right]} \sup _{x}\left|u_{n}^{q}(t, x)-u_{m}^{q}(t, x)\right|=0
$$

Теорема доказана.

Вернемся к рассмотрению исходной задачи, отказавшись от условия $a_{q}=b_{q}=c_{q}=0$. Пусть

$$
\widehat{\eta}_{u}^{q}(t)=\exp \left\{\int_{0}^{t}\left[a_{q}-b_{q} u^{1}\left(\theta, \widehat{\xi}^{q}(\theta)\right)-c_{q} u^{2}\left(\theta, \widehat{\xi}^{q}(\theta)\right)\right] d \theta\right\} .
$$

Обозначим $\widehat{\zeta}^{q}(t)=\left(\widehat{\xi}^{q}(t), \widehat{\eta}^{q}(t)\right), U^{q}(t, x)=\left(u^{q}(t, x), \nabla u^{q}(t, x)\right), V^{q}(t, x)=$ $\left(v^{q}(t, x), \nabla v^{q}(t, x)\right), U^{q}(0, x)=\left(u_{0 q}(x), \nabla u_{0 q}(x)\right)$ и пусть

$$
U_{V}^{q}(t, x)=\mathbf{E}\left[U_{0 q}\left(\widehat{\zeta}_{V}^{q}(t)\right)\right]=\left(\mathbf{E}\left[\eta_{v}^{q}(t) u_{0 q}\left(\widehat{\xi}_{v^{q}}^{q}(t)\right)\right], \mathbf{E}\left[Z_{V}^{q}\left(\widehat{\zeta}_{V}^{q}(t)\right)\right]\right)
$$

где $Z_{V}^{q}\left(\widehat{\zeta}^{q}(t)\right)=\nabla\left[\widehat{\eta}_{v}^{q}(t)\right] u_{0 q}\left(\widehat{\xi}_{v^{q}}^{q}(t)\right)+\widehat{\eta}_{v}^{q}(t) \nabla u_{0 q}\left(\widehat{\xi}_{v^{q}}^{q}(t)\right) \widehat{\mathbf{J}}_{v^{q}}^{q}(t)$.

Лемма 3.4. Пусть выполнены условия леммы 3.1 и $u_{0 q} \in H^{1}$ ограниченные функиии. Тогда для некоторой положительной константьл $T, 0 \leqslant t \leqslant T$, справедливь оценки

$$
\begin{gathered}
\sup _{x}\left|u_{v^{q}}^{q}(t, x)\right|^{2} \leqslant K_{0 q} e^{a_{q} t}, \\
\sup _{x}\left\|U_{V}^{q}(t, x)\right\|^{2} \leqslant\left\|M_{V}(t)\right\| \leqslant\left\|A_{V}(t)\right\|\left\|M_{0}\right\|,
\end{gathered}
$$


Д о к а з а т е л ь с т в о. Первая оценка непосредственно вытекает из формулы (3.16) с учетом условий на $u_{0 q}$ и $v^{q}$. Для доказательства второй оценки рассмотрим

$$
\begin{aligned}
U_{V}^{q}(t, z)=N^{q}(0, t) U_{0}^{q}(z) & =\mathbf{E}\left[U_{0}^{q}\left(\widehat{\zeta}^{q}(t)\right)\right] \\
& =\left(\mathbf{E}\left[\eta_{v}^{q}(t) u_{0 q}\left(\widehat{\xi}^{q}(t)\right), \mathbf{E}\left[Z_{V}\left(\widehat{\zeta}^{q}(t)\right)\right]\right) .\right.
\end{aligned}
$$

Обозначим

$$
\gamma_{v}^{q}(t)=\int_{0}^{t}\left[b_{q} \nabla v^{1}(\theta, \widehat{\xi}(\theta))+c_{q} \nabla v^{2}(\theta, \widehat{\xi}(\theta))\right] d \theta \mathbf{J}_{v}^{q}(\theta)
$$

и оценим

$$
\begin{aligned}
\sup _{x}\left\|U_{V}^{q}(t, x)\right\|^{2} & =\mathbf{E}\left\|\left(\begin{array}{cc}
\eta^{q}(t) & 0 \\
\gamma^{q}(t) \eta^{q}(t) & \eta^{q}(t) \mathbf{J}^{q}(t)
\end{array}\right)\left(\begin{array}{c}
u_{0}\left(\widehat{\xi}^{q}(t)\right) \\
\nabla u_{0}\left(\widehat{\xi}^{q}(t)\right)
\end{array}\right)\right\|^{2} \\
& \leqslant \mathbf{E}\left[\left|\eta^{q}(t)\right|^{2}\left\|\left(\begin{array}{cc}
1 & 0 \\
\gamma_{v}^{q}(t) & \mathbf{J}_{v}^{q}(t)
\end{array}\right)\right\|^{2}\right]\left\|U_{0}\right\|_{\infty}^{2} \\
& \leqslant e^{2 a_{q} t} K_{0}^{2} \mathbf{E}\left\|\left(\begin{array}{cc}
1 & 0 \\
\gamma_{v}^{q}(t) & \mathbf{J}_{v}^{q}(t)
\end{array}\right)\right\|^{2}
\end{aligned}
$$

Оценим математическое ожидание квадрата нормы Фробениуса матрицы $S^{q}(t)=\left(\begin{array}{cc}1 & 0 \\ \gamma_{v}^{q}(t) & \mathbf{J}^{q}(t)\end{array}\right)$.

$$
\mathbf{E}\left\|S^{q}(t)\right\|^{2}=1+\mathbf{E}\left\|\gamma_{v}^{q}(t)\right\|^{2}+\mathbf{E}\left\|\mathbf{J}_{v}^{q}(t)\right\|^{2},
$$

используя оценки

$$
\begin{aligned}
\mathbf{E}\left\|\gamma^{q}(t)\right\|^{2} & \leqslant t \mathbf{E}\left[\int_{0}^{t} 2\left[b_{q}\left\|\nabla v^{1}(\theta, \widehat{\xi}(\theta))\right\|^{2}+c_{q}\left\|\nabla v^{2}(\theta, \widehat{\xi}(\theta))\right\|\right] d \theta\left\|\mathbf{J}^{q}(t)\right\|^{2}\right] \\
& \leqslant t \int_{0}^{t} 2\left[b_{q} K_{v^{1}}^{1}(\theta)+c_{q} K_{v^{2}}^{1}(\theta)\right] d \theta \exp \left\{\int_{0}^{t} \Lambda_{q} K_{v^{q}}^{1}(\tau) d \tau\right\}
\end{aligned}
$$

и

$$
\mathbf{E}\left\|\mathbf{J}_{v^{q}}^{q}(t)\right\|^{2} \leqslant \exp \left\{\int_{0}^{t} \Lambda_{q} K_{v^{q}}^{1}(\tau) d \tau\right\} .
$$

Суммируя полученные оценки, придем к неравенству

$$
K_{U_{V}}^{1}(t) \leqslant K_{U_{V}^{q}}^{1}(0)\left[1+\exp \left\{\int_{0}^{t} G K_{v}^{1}(\tau) d \tau\right\}\right],
$$

где $G$ - константа, зависящая от параметров $a_{q}, \alpha_{q q}$ и $K_{v}^{1}(\tau)=K_{v^{1}}^{1}(\tau)+$ $K_{v^{2}}^{2}(\tau)$. Рассмотрим функцию $X(t), X(t)=2 K_{V}(0)\left[1+\exp \left\{\int_{0}^{t} G X(\tau) d \tau\right\}\right]$ и заметим, что $X(t)$ удовлетворяет следующей задаче Коши:

$$
\frac{d X(t)}{d t}=G X(0)\left[X^{2}(t)-X(t)\right], \quad X(0)=2 K_{V}(0)
$$


При этом, как нетрудно проверить, $X(t)$ имеет вид

$$
X(t)=\frac{2 K_{V}(0)}{2 K_{V}(0)-2 K_{V}(0) e^{2 K_{V}(0) G t}+e^{2 K_{V}(0) G t}}
$$

и является ограниченной функцией на интервале $[0, T]$, если $2 K_{V}(0)<1$. В противном случае $X(t)$ ограничена на интервале $\left[0, T_{1}\right]$ при условии

$$
T_{1}<\frac{1}{2 G K_{V}(0)} \ln \frac{2 K_{V}}{1-2 K_{V}} .
$$

Аналогично тому, как это было сделано при доказательстве теоремы 3.4, применяя, оценки леммы 3.5 к системе последовательных приближений, заданных соотношениями (3.10), (3.11) и

$$
\begin{aligned}
& u_{n}^{q}(t)= \mathbf{E}\left(\exp \left(\int_{0}^{t} m_{u_{n-1}}^{q}\left(\widehat{\xi}_{n-1}^{q}(\theta)\right) d \theta\right) u_{0}^{q}\left(\widehat{\xi}_{n-1}^{q}(t)\right)\right) \\
& \nabla u_{n}^{q}(t)=\mathbf{E}\left[\int_{0}^{t} \nabla m_{u_{n-1}^{1}, u_{n-1}^{2}}^{q}\left(\widehat{\xi}_{n-1}^{q}(t)\right) \widehat{\mathbf{J}}_{n-1}^{q}(\theta) d \theta\right. \\
&\left.\times \exp \left(\int_{0}^{t} m_{u_{n-1}^{1}, u_{n-1}^{2}}^{q}\left(\widehat{\xi}_{n-1}^{q}(t)\right) d \theta\right) u_{0}^{q}\left(\widehat{\xi}_{n-1}^{q}(t)\right)\right] \\
&+\mathbf{E}\left[\exp \left(\int_{0}^{t} m_{u_{n-1}^{1}, u_{n-1}^{2}}^{q}\left(\widehat{\xi}_{n-1}^{q}(t)\right) d \theta\right) \nabla u_{0}^{q}\left(\widehat{\xi}_{n-1}^{q}(t)\right) \widehat{\mathbf{J}}_{n-1}^{q}(t)\right]
\end{aligned}
$$

можно показать, что справедливо следующее утверждение.

Теорема 3.2. Пусть выполнень условия леммь 3.4. Тогда процессыл $\widehat{\xi}_{n}^{q}(t), \widehat{\mathbf{J}}_{n}^{q}(t)$, удовлетворяющие (3.10), (3.11), и функиии $u_{n}^{q}(t), \nabla u_{n}^{q}(t)$, удовлетворяюшие (3.31)-(3.32), сходятся в соответствуюших нормах к решению системь (3.5), (3.6), (3.8), (3.9).

Как уже упоминалось выше, после того, как доказано существование решения стохастической системы (3.4)-(3.7), мы снова оказываемся в ситуации, когда нелинейность рассматриваемой задачи не имеет значения. Другими словами мы снова можем воспользоваться результатами, вытекающими из теоремы 2.1 работы [12].

Покажем, наконец, что построенные в этом пункте решения стохастической системы (3.4)-(3.7) приводят к построению обобщенного решения исходной задачи Коши (1.1), (1.2) в отсутствие кросс-диффузии.

Теорема 3.3. Пусть $u_{0}^{q} \in H^{2}\left(\mathbf{R}^{d}\right)$ - заданные ограниченные функ-

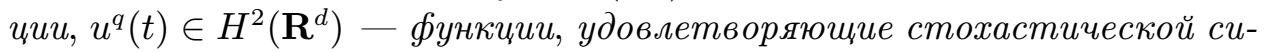
cтеме (3.2)-(3.4), (3.7) u

$$
\kappa^{q}(t)=\exp \left(\int_{0}^{t} m_{u}^{q} \circ \phi_{0, \theta} d \theta\right) u_{0}^{q} .
$$


Тогда функиии $u^{q}(t)=\mathbf{E}\left[\kappa^{q}(t) \circ \psi_{t, 0}^{q}\right], q=1,2$, удовлетворяют в обобшенном смилсле задаче Коши

$$
u_{t}^{q}=\mathscr{L}_{u}^{q} u^{q}+m_{u}^{q} u^{q}, \quad u^{q}(0)=u_{0}^{q}
$$

при $\alpha_{12}=\alpha_{21}=0$ и существуют положительнье константьл $C^{q}$, зависящие только от $T$ и $\mathscr{L}^{q}$, для которых справедливы оченки

$$
\left\|u^{q}(t)\right\|_{1}^{2} \leqslant C^{q}\left\|u_{0}^{q}\right\|_{1}^{2} \quad \forall t \in[0, T] .
$$

Д ок а з а т е ль с т в о. Если $u_{0} \in H^{1}$, то $\kappa^{q}(t)-$ непрерывный семимартингал. Поскольку $\kappa^{q}(t)$ удовлетворяет ОДУ

$$
\frac{d \kappa^{q}(t)}{d t}=m_{u}^{q} \kappa^{q}(t), \quad \kappa^{q}(0)=u_{0}^{q}
$$

то из обобщенной формулы Ито следует, что

$$
\begin{aligned}
\kappa^{q}(t) \circ \psi_{0, t}^{q}= & u_{0}^{q}+\int_{0}^{t} \frac{\partial \kappa^{q}(\theta)}{\partial \theta} \circ \psi_{\theta, t}^{q} d \theta+\int_{0}^{t} \nabla \kappa^{q}(\theta) \circ \psi_{\theta, t}^{q} \cdot d \psi^{q}(\theta) \\
& +\int_{0}^{t} \mathscr{L}_{u}^{q}\left(\kappa^{q}(\theta) \circ \psi_{\theta, t}^{q}\right) d \theta
\end{aligned}
$$

или, с учетом (3.36),

$$
\begin{aligned}
\kappa^{q}(t) \circ \psi_{0, t}^{q}= & u_{0}^{q}+\int_{0}^{t} \nabla\left(\kappa^{q}(t) \circ \psi_{\theta, t}^{q}\right) M_{u}^{q}(x) \cdot d w(\theta) \\
& +\int_{0}^{t} \mathscr{L}_{u}^{q}\left(\kappa^{q}(\theta) \circ \psi_{\theta, t}^{q}\right) d \theta
\end{aligned}
$$

Дальнейшие рассуждения совпадают с рассуждениями, приведенными при доказательстве теоремы 2.2. Вычислим математическое ожидание обеих частей соотношения (3.38). Поскольку

$$
\mathbf{E}\left\langle\int_{0}^{t} \nabla\left(\kappa^{q}(t) \circ \psi_{\theta, t}^{q}\right), M_{u}^{q}(x) d w(\theta), h\right\rangle=0,
$$

И

$$
\begin{gathered}
\left.\mathbf{E}\left[\left\langle\int_{0}^{t} \mathscr{L}_{u}^{q}\left[\kappa^{q}(\theta) \circ \psi_{\theta, t}^{q}\right)\right] d \theta, h\right\rangle\right]=\mathbf{E}\left[\int_{0}^{t}\left\langle\kappa^{q}(\theta) \circ \psi_{\theta, t}^{q}, \mathscr{M}_{u}^{q} h\right\rangle d \theta\right] \\
=\int_{0}^{t}\left\langle\mathbf{E}\left[\kappa^{q}(\theta) \circ \psi_{\theta, t}^{q}\right],\left[\frac{1}{2}\left[M_{u}^{q}\right]^{2} \Delta h+m_{u}^{q} h\right]\right\rangle d \theta \\
=\int_{0}^{t}\left\langle\mathscr{L}_{u}^{q} \mathbf{E}\left[\kappa^{q}(\theta) \circ \psi_{\theta, t}^{q}\right], h\right\rangle d \theta
\end{gathered}
$$

то получим равенство

$$
\mathbf{E}\left[\kappa^{q}(t) \circ \psi_{0, t}^{q}\right]=u_{0}^{q}+\int_{0}^{t}\left\langle\left[\mathscr{L}_{u}^{q}\right] \mathbf{E}\left[\kappa^{q}(\theta) \circ \psi_{\theta, t}^{q}\right], h\right\rangle d \theta .
$$


Вычисляя производную по переменной $t$ от обеих частей соотношения (3.40), получим, что функции $u^{q}(t)=\mathbf{E}\left[\kappa^{q}(t) \circ \psi_{0, t}^{q}\right]$ удовлетворяют задаче Коши (3.35), поскольку $\lim _{t \rightarrow 0}\left\langle u^{q}(t), h\right\rangle=\left\langle u_{0}^{q}, h\right\rangle$ и, следовательно, $\lim _{t \rightarrow 0} u^{q}(t)=u_{0}^{q}$. Теорема доказана.

3 а м е ч а н и е. Как следует из результатов предыдущего пункта, функции $u^{q}(t)$, удовлетворяющие системе (3.4)-(3.7), — это дифференцируемые функции. Для того, чтобы показать, что построенное выше решение задачи (3.35) единственно, предположим обратное, т.е. что существует два регулярных обобщенных решения $\left(u^{1}, u^{2}\right)$ и $\left(\widetilde{u}^{1}, \widetilde{u}^{2}\right)$ этой задачи. Тогда их разность $R^{q}(t)=u^{q}(t)-\widetilde{u}^{q}(t)$ удовлетворяет уравнению $\partial R^{q}(t) / \partial t=\mathscr{L}_{u}^{q} R^{q}(t)$ и условию $R^{q}(0)=0$, откуда следует, что $R^{q}(t)=0$. Для того, чтобы это показать, заметим, что для любого $t>0$ и $h \in C_{0}^{2} \cap H^{2}$ существует решение $h^{q}(s, y)$ параболического уравнения $h_{s}^{q}+\frac{1}{2}\left[M_{u}^{q}\right]^{2} \Delta h+m_{u}^{q} h^{q}=0$, удовлетворяющее условию $h^{q}(t)=h(y)$, и при этом $h^{q}(s)$ принадлежит $C^{2}\left(\mathbf{R}^{d}\right)$. Отсюда вытекает, что для любой функции $h \in C_{0}^{2} \cap H^{2}$ справедливы соотношения

$$
\begin{aligned}
\left\langle R^{q}(t), h\right\rangle= & \int_{0}^{t}\left\langle\frac{d}{d \theta} R^{q}(\theta), h(\theta)\right\rangle d \theta+\int_{0}^{t}\left\langle R^{q}(\theta), \frac{\partial h(\theta)}{\partial \theta}\right\rangle d \theta \\
= & \int_{0}^{t}\left\langle\mathscr{L}_{u}^{q} R^{q}(\theta), h(\theta)\right\rangle d \theta \\
& -\int_{0}^{t}\left\langle R^{q}(\theta),\left[\frac{1}{2}\left[M_{u}^{q}\right]^{2} \Delta h+m_{u}^{q} h(\theta)\right]\right\rangle d \theta=0,
\end{aligned}
$$

следовательно, $R^{q}(t)=0$.

\section{4. Вероятностный подход к построению обобщенных ре-} шений задачи Коши для полностью недиагональных параболических систем. В этом пункте мы рассмотрим идеи, лежащие в основе вероятностного подхода к построению обобщенных решений задачи Коши для полностью недиагональных параболических систем. Как и выше, мы рассмотрим стохастические уравнения, вид которых диктуется соотношением (1.3). При этом если пара функций $u$ и $h$ принадлежит соболевскому пространству $H^{k}$, то $\langle u, h\rangle=\int_{\mathbf{R}^{d}} u(x), h(x) d x$ будет обозначать соответствующее скалярное произведение.

Рассмотрим задачу Коши (1.1), (1.2), отказавшись от упрощающего предположения $\alpha_{12}=\alpha_{21}=0$, и построим вероятностное представление ее обобщенного решения $u=\left(u^{1}, u^{2}\right)$. Заметим, что в этом случае для получения требуемого результата не удается применить обобщенную формулу Ито к искомым функциям $u^{q}(s, x)$, что усложняет рассматриваемую задачу.

Для того, чтобы построить вероятностное представление обобщенного решения задачи Коши (1.1), (1.2) для системы с кросс-диффузией, предположим что существует неотрицательное регулярное обобщенное 
решение этой задачи. Как и в предыдущих пунктах, рассмотрим базовый случайный процесс $\xi_{s, \kappa}^{q}(\theta)$, удовлетворяющий СДУ

$$
d \xi_{0, \kappa}^{q}(\theta)=-M_{u}^{q}\left(\xi_{0, \kappa}^{q}(\theta)\right) d w(\theta), \quad \xi_{s, \kappa}^{q}(0)=\kappa,
$$

где $M_{u}^{q}=\sqrt{\alpha_{q}+\alpha_{q 1} u^{1}+\alpha_{q 2} u^{2}}$ и обозначим $J^{q}(t)$ якобиан преобразования $\phi_{0, t}: \kappa \rightarrow \phi_{0, t}(\kappa)=\xi_{0, \kappa}^{q}(t)$. Наряду с этим рассмотрим случайный процесс $\eta^{q}(\theta)$, удовлетворяющий линейному СДУ

$$
d \eta^{q}(\theta)=\widetilde{m}_{u}^{q}\left(\xi_{s, \kappa}^{q}(\theta)\right) \eta^{q}(\theta) d \theta+C_{u}^{q}\left(\xi_{s, \kappa}^{q}(\theta)\right) \eta^{q}(\theta) d w(\theta), \quad \eta^{q}(0)=1,
$$

коэффициенты $\widetilde{m}_{u}^{q}$ и $C_{u}^{q}$ которого будут выбраны ниже.

Рассмотрим случайный процесс

$$
r_{\kappa}^{q}(t)=\eta^{q}(t) h\left(\xi_{0, \kappa}^{q}(t)\right) J^{q}(t)
$$

и заметим, что этот процесс порождает семейство ограниченных линейных отображений $K^{q}(t)$, действующих в пространстве $H^{1}\left(\mathbf{R}^{d}\right)$, заданное соотношением

$$
\mathbf{E}\left[r_{\kappa}^{q}(t)\right]=K^{q}(t) h(\kappa),
$$

и обладающее полугрупповым свойством, т.е. $K^{q}(t) K^{q}(\theta) h=K^{q}(t+\theta) h$.

В этом пункте мы отождествляем гильбертово пространство $H^{k}, k=0,1,2$ с двойственным и под спариванием $\langle u, h\rangle$ подразумеваем скалярное произведение $u$ и $h$ в $H^{k}$.

Пусть $u^{q} \in \mathscr{W}^{1}$ - ограниченные положительные функции, удовлетворяющие системе (1.1), (1.2) в смысле интегральных тождеств (0.3). Тогда из общих результатов теории стохастических уравнений следует, что существуют процессы $\xi^{q}(t)$, удовлетворяющие СДУ (4.1), и процессы $\eta^{q}(t)$, удовлетворяющие СДУ (4.2) при соответствующем выборе коэффициентов. Поскольку, как будет показано ниже, при $q=1,2$ процессы $J^{q}(t)$ также удовлетворяют соответствующим линейным СДУ, то из единственности решений линейных СДУ и марковского свойства процесса $\xi^{q}(t)$ нетрудно вывести, что семейство $K^{q}(t)$ обладает полугрупповым свойством.

При $q=1,2$ нас будет интересовать семейство отображений $\left[K^{q}\right]^{+}(t)$, сопряженное к семейству $K^{q}(t)$, т.е. семейство

$$
\left\langle\left[K^{q}\right]^{+}(t) u, h\right\rangle=\left\langle u, K^{q}(t) h\right\rangle \quad \forall u, h \in H^{1},
$$

и его вероятностное представление. Для того, чтобы построить вероятностное представление этого семейства наряду с процессами $\xi^{q}(t), \eta^{q}(t)$, удовлетворяющими (4.1), (4.2), введем в рассмотрение случайные процессы $\zeta^{q}(t)$ вида

$$
\zeta^{q}(t)=\exp \left\{\int_{0}^{t} n_{u}^{q}\left(\phi_{0, \theta}^{q}\right) d \theta+\int_{0}^{t} C_{u}^{q}\left(\phi_{0, \theta}^{q}\right) d w(\theta)\right\} u_{0}^{q}
$$


И

$$
\zeta^{q}(t) \circ \psi_{0, t}=\exp \left\{\int_{0}^{t} n_{u}^{q}\left(\psi_{\theta, t}^{q}\right) d \theta+\int_{0}^{t} C_{u}^{q}\left(\psi_{\theta, t}^{q}\right) d w(\theta)\right\} u_{0}^{q} \circ \psi_{0, t},
$$

где $n_{u}^{q}=\widetilde{m}_{u}^{q}-\frac{1}{2}\left\|C_{u}^{q}\right\|^{2}$.

Пусть $u_{0}^{q} \in H^{k}, k \geqslant 1$ тогда, как следует из теории стохастических потоков, процесс $\zeta^{q}(t)$ - это процесс в пространстве $H^{k}$ и его композиция с потоком $\psi_{0, t}^{q}$ также представляет собой случайный процесс в $H^{k}$.

Из теоремы 2.1 работы [11] следует, что существуют константы $C_{q, k}$, для которых справедливы следующие оценки

$$
\mathbf{E}\left\|\zeta^{q}(t) \circ \psi_{0, t}^{q}\right\|_{k}^{2} \leqslant C_{q, k}\left\|u_{0}^{q}\right\|_{k}^{2}
$$

если $u_{0}^{q} \in H^{k}$ - ограниченные функции.

Как вытекает из этих оценок, величина

$$
\left\langle\Lambda_{0, t}^{q}, h\right\rangle=\mathbf{E}\left[\left\langle\zeta^{q}(t) \circ \psi_{0, t}^{q}, h\right\rangle\right]
$$

корректно определена для любых $h \in H^{1}$ и определяет линейный непрерывный функционал на двойственном пространстве. В дальнейшем мы будем обозначать $\Lambda^{q}(t)=\mathbf{E}\left[\zeta^{q}(t) \circ \psi_{0, t}^{q}\right]$.

Выберем коэффициенты $\widetilde{m}_{u}^{q}, C_{u}^{q}$ в уравнении (4.2) так, чтобы производящий оператор $\mathscr{M}^{q}$ семейства $K^{q}(t)$ имел вид

$$
\mathscr{M}^{q} h=\frac{1}{2} M_{u}^{q} \Delta h+m_{u}^{q} h
$$

и покажем, что при таком выборе коэффициентов $\tilde{m}_{u}^{q}, C_{u}^{q}$ регулярное обобщенное решение $u=\left(u^{1}, u^{2}\right)$ задачи Коши для полной системы (1.1), (1.2) допускает стохастическое представление вида

$$
u^{q}(t)=\mathbf{E}\left[\zeta^{q}(t) \circ \psi_{0, t}^{q}\right]
$$

где процесс $\zeta^{q}(t)$ задан соотношением (4.6), а $\psi_{0, t}^{q}-$ стохастический поток, обратный по отношению к потоку $\phi_{0, t}^{q}$, порожденному решением СДУ (4.1).

Иначе говоря, покажем, что справедливо следующее утверждение.

Теорема 4.1. Пусть $u^{1}, u^{2} \in \mathscr{W}^{1}$ - обобщенное решение системь (1.1), (1.2) и коэффиииенть $\widetilde{m}_{u}^{q}, C_{u}^{q}$ имеют вид

$$
\begin{gathered}
\tilde{m}_{u}^{q}=m_{u}^{q}-\left\|\nabla M_{u}^{q}\right\|^{2}=m_{u}^{q}-\frac{\left\|\alpha_{q 1} \nabla u^{1}+\alpha_{q 2} \nabla u^{2}\right\|^{2}}{4\left[\alpha_{q}+\alpha_{q 1} u^{1}+\alpha_{q 2} u^{2}\right]}, \\
C_{u}^{q}=-\nabla M_{u}^{q}=-\frac{\left[\alpha_{q 1} \nabla u^{1}+\alpha_{q 2} \nabla u^{2}\right]}{2 \sqrt{\left[\alpha_{q}+\alpha_{q 1} u^{1}+\alpha_{q 2} u^{2}\right]}} .
\end{gathered}
$$


Тогда функиии $u^{q}(t)$ допускают вероятностное представление вида (4.9), где случайнье прочессьь $\zeta^{q}(t)$ задань соотношениями (4.6), а $\psi_{0, t}^{q}$ - обратные потоки по отношению $\kappa$ потокам $\phi_{0, t}^{q}$, порожденным решениями $\xi^{q}(t)$ СДУ (4.1).

Д о к а з а т е л ь с т в о этой теоремы разобьем на несколько отдельных утверждений.

Пусть $u_{0}^{q}$ - заданный элемент из $H^{1}$. Тогда корректно определен случайный процесс $\left\langle u_{0} \circ \psi_{0, t}, h\right\rangle$ с ограниченными вторыми моментами. Пусть процесс $\zeta^{q}(t)$ задан соотношением (4.6). Тогда пара процессов $\left(\psi_{0, t}^{q}, \zeta^{q}(t)\right)$ образует двухкомпонентный марковский процесс. При этом $\left\langle U_{0, t}^{q}, h\right\rangle \equiv \mathbf{E}\left[\left\langle\zeta^{q}(t) u_{0}^{q} \circ \psi_{0, t}^{q}, h\right\rangle\right]$ - это непрерывный линейный функционал на $H^{1}$, поэтому его можно рассматривать как элемент сопряженного пространства. Этот элемент называют обобщенным математическим ожиданием величины $\gamma_{0, t}^{q}=\zeta^{q}(t) u_{0}^{q} \circ \psi_{0, t}^{q}$, т.е.

$$
\left[K_{t}^{q}\right]^{+} u_{0}^{q}=\mathbf{E}\left[\gamma_{0, t}^{q}\right]
$$

Заметим, что $\left[K^{q}\right]_{t}^{+}-$это линейное отображение, действующее в пространстве $H^{1}$ и обладающее полугрупповым свойством, т.е. $\left[K^{q}\right]_{t}^{+}\left[K^{q}\right]_{\theta}^{+}=\left[K_{t+\theta}^{q}\right]^{+}$для любых $t, \theta>0$. Действительно,

$$
\begin{aligned}
\left\langle\left[K^{q}\right]_{t}^{+}\left[K^{q}\right]_{\theta}^{+} u_{0}^{q}, h\right\rangle & \left.=\mathbf{E}\left[\left[K^{q}\right]_{\theta}^{+} u_{0},\left[\eta^{q}(t) h \circ \phi_{0, t}^{q}\right] J_{0, t}^{q}\right\rangle\right] \\
& =\mathbf{E}\left[u_{0}^{q},\left.\eta^{q}(t+\theta) g \circ \phi_{t, t+\theta} J_{t, t+\theta}^{q}\right|_{\left.g=\eta^{q}(t) h \circ \phi_{0, t}^{q} J_{0, t}^{q}\right]}\right] \\
& =\mathbf{E}\left[\left\langle u_{0}, \eta^{q}(t+\theta) h \circ \phi_{0, t}^{q} \circ \phi_{t, t+\theta}^{q}\right)\left[J_{0, t}^{q} J_{0, t+\theta}^{q}\right]\right. \\
& =\mathbf{E}\left[\left\langle K_{0, t+\theta}^{q} u_{0}^{q}, h\right\rangle\right] .
\end{aligned}
$$

Функции $u^{q}(t)$ корректно определены соотношениями (4.7). Покажем, что они удовлетворяют соотношению (0.3) для любой функции $h \in H^{1}$, т.е. являются обобщенными решениями рассматриваемой системы.

Вычислим стохастический дифференциал процесса $r^{q}(\theta)$, заданного соотношением

$$
r^{q}(\theta) \equiv r_{0}^{q}(\theta, \kappa)=\eta^{q}(\theta) h\left(\xi_{0, \kappa}^{q}(\theta)\right) J^{q}\left(\xi_{0, \kappa}^{q}(\theta)\right) .
$$

Напомним, что $J^{q}(\theta)=J^{q}\left(\xi_{s, \kappa}^{q}(\theta)\right)$ - это якобиан преобразования $\kappa \rightarrow \phi_{0, \theta}^{q}(\kappa)$, порожденного процессом $\xi^{q}(\theta)$, удовлетворяющим СДУ $(4.1)$, т.е. $J^{q}(\theta)=\operatorname{det} \nabla \phi_{0, \theta}^{q}(\kappa)$. При вычислении стохастического дифференциала процесса $r^{q}(\theta)$ нам понадобится явное выражение для стохастического дифференциала якобиана $J^{q}(\theta)$.

Лемма 4.1. Пусть $\mathbf{J}^{q}(t)$ - якобиева матрица преобразования $\phi_{0, t}^{q}$, порожденного решением $\xi^{q}(t)$ СДУ (4.1). Тогда якобиан $J^{q}(t)=$ $\operatorname{det} \mathbf{J}^{q}(t)$ удовлетворяет СДУ

$$
d J^{q}(\theta)=J^{q}(\theta) \operatorname{Tr}\left(\widehat{\mathbf{J}}^{q}(\theta) d \mathbf{J}^{q}(\theta)\right), \quad J^{q}(0)=1 .
$$


Д о к а з а т е л ь с т в о. Пусть $G(\theta)$ - неслучайная матрица, тогда ее детерминант $\operatorname{det} G$ удовлетворяет обыкновенному дифференциальному уравнению

$$
\frac{d}{d \theta} \operatorname{det} G(\theta)=\operatorname{det} G(\theta) \operatorname{Tr}\left[G^{-1}(\theta) \frac{d}{d \theta} G(\theta)\right] .
$$

При выводе этого уравнения важную роль играет тот факт, что детерминант $\operatorname{det} G$ является полилинейной функцией строк матрицы $G$, т.е. $\operatorname{det} G(t)=l\left(g_{1}(t), \ldots, g_{d}(t)\right)$, где $l$ - линейная функция аргумента $g_{k}(t), k=1, \ldots, d$, при фиксированных остальных аргументах и, следовательно,

$$
\frac{d}{d \theta} \operatorname{det} G(\theta)=\sum_{k=1}^{d} l\left(g_{1}(\theta), \ldots, \frac{d}{d \theta} g_{k}(\theta), \ldots, g_{d}(\theta)\right)
$$

Если же $G(\theta)$ — обратимая стохастическая матрица, имеющая стохастический дифференциал $d G(\theta)$, то, в силу полилинейности функции det, нетрудно проверить, что последнее соотношение приобретает вид

$$
d[\operatorname{det} G(\theta)]=\operatorname{det} G(\theta) \operatorname{Tr}\left[G^{-1}(\theta) d G(\theta)\right] .
$$

(Напомним, что для линейных отображений формула Ито не содержит поправочных слагаемых.)

Поскольку в рассматриваемых условиях матрица $\mathbf{J}^{q}(\theta)=\nabla \phi_{0, \theta}^{q}$ обратима и ее стохастический дифференциал $d \mathbf{J}^{q}$ имеет вид $(2.14)$, то отсюда следует, что $J^{q}(\theta)$ удовлетворяет (4.13). Лемма доказана.

Пусть

$$
\mathscr{M}_{u}^{q} h=\frac{1}{2}\left[M_{u}^{q}\right]^{2} \Delta h+m_{u}^{q} h
$$

- оператор сопряженный в $H^{1}$ к оператору $\mathscr{L}_{u}^{q}$ вида (1.5). Воспользуемся классической формулой Ито для вычисления стохастического дифференциала процесса $r^{q}(\theta)$.

Лемма 4.2. Пусть коэффициенть в линейном СДУ задань соотношениями (4.10). Тогда справедливы соотношения

$$
d r_{0}^{q}(\theta, \kappa)=\left[\mathscr{M}_{u}^{q} h\right]\left(\xi_{0, \kappa}^{q}(\theta)\right) \eta^{q}(\theta) J^{q}(\theta) d \theta+\eta^{q}(\theta) J^{q}(\theta) N_{u}^{q}(\theta) d w(\theta),
$$

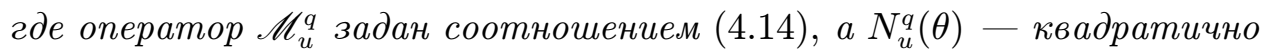
интегрируемьй прочесс, $и$

$$
\mathbf{E}\left[r_{0}^{q}(t, \kappa)\right]=h(\kappa)+\mathbf{E}\left[\int_{0}^{t}\left[\mathscr{M}_{u}^{q} h\right]\left(\xi_{0, \kappa}^{q}(\theta)\right) \eta^{q}(\theta) J^{q}(\theta) d \theta\right] .
$$


Д о к а з а т е л ь с т в о. Применив формулу Ито, получим

$$
\begin{aligned}
d r^{q}(\theta)= & d\left[\eta^{q}(\theta) h\left(\xi_{0, \kappa}^{q}(\theta)\right) J^{q}(\theta)\right] \\
= & d\left[\eta^{q}(\theta)\right] h\left(\xi_{0, \kappa}^{q}(\theta)\right) J^{q}(\theta)+\eta^{q}(\theta) d\left[h\left(\xi_{0, \kappa}^{q}(\theta)\right)\right] J^{q}(\theta) \\
& +h^{q}\left(\xi_{0, \kappa}^{q}(\theta)\right) d\left[J^{q}(\theta)\right]+d\left[\eta^{q}(\theta)\right] d\left[h\left(\xi_{0, \kappa}^{q}(\theta)\right)\right] J^{q}(\theta) \\
& +\eta^{q}(\theta) d\left[h\left(\xi_{0, \kappa}^{q}(\theta)\right)\right] d\left[J^{q}(\theta)\right]+d\left[\eta^{q}(\theta)\right] h\left(\xi_{0, \kappa}^{q}(\theta)\right) d\left[J^{q}(\theta)\right] .
\end{aligned}
$$

С учетом явного вида стохастических дифференциалов $d \eta^{q}(\theta), d h\left(\xi_{0, \kappa}^{q}(\theta)\right)$ и $d J^{q}(\theta)$ нетрудно показать, что справедливо соотношение

$$
\begin{aligned}
d r^{q}(\theta)= & {\left[\left[\mathscr{L}_{0}^{q}(u)\right]^{*} h\left(\xi_{0, \kappa}^{q}(\theta)\right)+\widetilde{m}_{u}^{q}\left(\xi_{0, \kappa}^{q}(t)\right) h\left(\xi_{0, \kappa}^{q}(\theta)\right)\right.} \\
& +\left[C_{u}^{q} M_{u}^{q}\right]\left(\xi_{0, \kappa}^{q}(\theta) \nabla h\left(\xi_{0, \kappa}^{q}(\theta)\right)\right] J^{q}(\theta) \eta^{q}(\theta) d \theta \\
- & {\left[\left[C_{u}^{q} \cdot \nabla M_{u}^{q}\right]\left(\xi_{0, \kappa}^{q}(\theta)\right) h\left(\xi_{0, \kappa}^{q}(\theta)\right)\right.} \\
& \left.+\left[\nabla M_{u}^{q}\left(\xi_{0, \kappa}^{q}(\theta)\right)\right] M_{u}^{q}\left(\xi_{0, \kappa}^{q}(\theta)\right) \nabla h\left(\xi_{0, \kappa}^{q}(\theta)\right)\right] \eta^{q}(\theta) J^{q}(\theta) d \theta \\
+ & J^{q}(\theta) \eta^{q}(\theta) N_{u}^{q}(\theta) d w(\theta),
\end{aligned}
$$

где $N_{u}^{q}(\theta)=M_{u}^{q} \nabla h\left(\xi_{0, \kappa}^{q}(\theta)\right)+\left[C_{u}^{q}+\nabla M_{u}^{q}\right] h\left(\xi_{0, \kappa}^{q}(\theta)\right)$. Анализируя (4.17), можно заметить, что для любой вещественной функции $h \in C_{c}^{3}\left(R^{n}\right)$ и $0 \leqslant s \leqslant t \leqslant T$, процессы $r_{0}^{q}(\theta)$ имеют стохастические дифференциалы вида (4.15). Интегрируя последнее соотношение в пределах от 0 до $t$ и вычисляя математическое ожидание, получим равенство (4.16). Лемма доказана.

Д ок аз а т ель с т в о т е о р е м 4.1. Как и в предыдущих пунктах, для заданной функции $v \in H^{k}$ случайный процесс $V^{q}(t)=$ $v \circ \psi_{0, t}^{q}$ определенный с помощью соотношения

$$
\left\langle v \circ \psi_{0, t}^{q}, h\right\rangle=\left\langle v, h \circ \phi_{0, t}^{q} J^{q}(t)\right\rangle
$$

будет рассматриваться как случайный процесс в $H^{k}$.

Рассмотрим случайный процесс $\zeta^{q}(t) \in H^{-1}$, заданный с помощью соотношения

$$
\left\langle\zeta^{q}(t) \circ \psi_{0, t}^{q}, h\right\rangle=\left\langle u_{0}^{q}, \eta^{q}(t) h \circ \phi_{0, t}^{q} J^{q}(t)\right\rangle=\left\langle u_{0}^{q}, r_{0}^{q}(t)\right\rangle .
$$

Пусть $u^{q}(t) \in H^{1}$ - обобщенное регулярное решение системы (1.1), (1.2). Тогда, как было показано выше, процессы $\eta^{q}(t)$ и $\xi^{q}(t)$ удовлетворяющие СДУ (4.1), (4.2), существуют и определены однозначно. При этом корректность (4.19) гарантируется оценками, приведенными в начале этого пункта. Заметим, что

$$
\mathbf{E}\left[\int_{\mathbf{R}^{d}} \int_{0}^{t} u_{0}^{q}(\kappa) d r^{q}(\theta) d \kappa\right]=\mathbf{E}\left[\int_{\mathbf{R}^{d}} u_{0}^{q}(\kappa) r^{q}(t) d \kappa\right]-\int_{\mathbf{R}^{d}} u_{0}^{q}(\kappa) h(\kappa) d \kappa,
$$


и при этом из соотношения (4.15) вытекает равенство

$$
\begin{aligned}
& \mathbf{E}\left[\int_{\mathbf{R}^{d}} \int_{0}^{t} u_{0}^{q}(\kappa) d r^{q}(\theta) d \kappa\right] \\
& \quad=\mathbf{E}\left[\int_{0}^{t} \int_{\mathbf{R}^{d}} u_{0}^{q}(\kappa) d\left[\eta^{q}(\theta) h\left(\xi_{0, \kappa}^{q}(\theta)\right) J^{q}\left(\xi_{0, \kappa}^{q}(\theta)\right)\right] d \kappa\right] \\
& =\mathbf{E}\left[\int_{0}^{t} \int_{\mathbf{R}^{d}} u_{0}^{q}(\kappa)\left[\mathscr{M}_{u}^{q}\right] h\left(\xi_{0, \kappa}^{q}(\theta)\right) \eta^{q}(\theta) J^{q}\left(\xi_{0, \kappa}^{q}(\theta)\right) d \kappa d \theta\right]
\end{aligned}
$$

Введем замену переменных $\xi_{0, \kappa}^{q}(\theta)=x$ и, используя стохастическую теорему Фубини, перепишем (4.21) в виде

$$
\begin{aligned}
\mathbf{E}\left[\int_{\mathbf{R}^{d}} \int_{0}^{t} u_{0}^{q}(\kappa) d r^{q}(\theta) d \kappa\right] & =\mathbf{E}\left[\int_{0}^{t} \int_{\mathbf{R}^{d}}\left[\widehat{\eta}^{q}(\theta) u_{0}^{q}\left(\widehat{\xi}_{0, x}^{q}(\theta)\right)\right]\left[\mathscr{M}_{u}^{q}\right] h(x) d x d \theta\right] \\
& =\int_{0}^{t} \int_{\mathbf{R}^{d}} \mathbf{E}\left[\widehat{\eta}^{q}(\theta) u_{0}^{q}\left(\widehat{\xi}_{0, x}^{q}(\theta)\right)\right] \mathscr{M}_{u}^{q} h(x) d x d \theta \\
& =\int_{0}^{t} \int_{\mathbf{R}^{d}} \mathscr{L}_{u}^{q} \mathbf{E}\left[\widehat{\eta}^{q}(\theta) u_{0}^{q}\left(\widehat{\xi}_{0, x}^{q}(\theta)\right)\right] h(x) d x d \theta
\end{aligned}
$$

а правую часть равенства (4.20) перепишем в виде

$$
\begin{aligned}
& \mathbf{E}\left[\int_{\mathbf{R}^{d}} u_{0}^{q}(\kappa) r^{q}(t) d \kappa\right]-\int_{\mathbf{R}^{d}} u_{0}^{q}(\kappa) r^{q}(0, \kappa) d \kappa \\
& \quad=\mathbf{E}\left[\int_{\mathbf{R}^{d}} \widehat{\eta}^{q}(t) u_{0}^{q}\left(\widehat{\xi}_{0, x}(t)\right) h(x) d x\right]-\int_{\mathbf{R}^{d}} u_{0}^{q}(x) h(x) d x .
\end{aligned}
$$

Сопоставляя соотношения (4.20)-(4.23), получим равенство

$$
\begin{aligned}
\mathbf{E} & {\left[\int_{\mathbf{R}^{d}} \mathbf{E}\left[\widehat{\eta}^{q}(t) u_{0}^{q}\left(\widehat{\xi}_{0, x}(t)\right)\right] h(x) d x\right] } \\
& =\int_{\mathbf{R}^{d}} u_{0}^{q}(x) h(x) d x+\int_{0}^{t} \int_{\mathbf{R}^{d}} \mathscr{L}_{u}^{q}\left(\mathbf{E}\left[\widehat{\eta}^{q}(\theta) u_{0}^{q}\left(\widehat{\xi}_{0, x}^{q}(\theta)\right)\right]\right) h(x) d x d \theta .
\end{aligned}
$$

Дифференцируя полученное соотношение (4.24) по $t$ и, принимая во внимание тот факт, что это равенство справедливо при любом $h \in H_{0}^{2}$, получим, что функции $u^{q}(t)=\mathbf{E}\left[\zeta^{q}(t) \circ \psi^{q}(t)\right]$ удовлетворяют соотношениям (1.1), (1.2) в смысле теории обобщенных функций.

Справедливость начального условия очевидна.

\section{СПИСОК ЛИТЕРАТУРЫ}

1. Amann H. Dynamic theory of quasilinear parabolic equations. II. Reaction-diffusion systems. - Diff. Int. Eqs., 1990, v. 3, p. 13-75. 
2. Mimura M., Kawasaki K. Spatial segregation in competitive interaction-diffusion equations. - J. Math. Biol., 1980, v. 9, p. 49-64.

3. Kim J. Smooth solutions to a quasi-linear system of diffusion equations for a certain population model. - Nonlin. Anal., 1984, v. 8, p. 1121-1144.

4. Jüngel $A$. Diffusive and nondiffusive population models. In: G. Naldi, L. Pareschi, and G. Toscani (eds.). Mathematical Modeling of Collective Behavior in Socio-Economic and Life Sciences. Birkhäuser, Basel, (2010), p. 397-425.

5. Desvillettes L., Lepoutre T., Moussa A. Entropy, Duality, and Cross Diffusion. SIAM J. Math. Analysis, 2014, v. 46, № 1, p. 820-853.

6. Shigesada N., Kawasaki K., Teramoto E. Spatial segregation of interacting species. J. Theor. Biol., 1979, v. 79, p. 83-99.

7. Belopolskaya Ya. Markov processes associated with fully nondiagonal systems of parabolic equations. - Markov Process. Related Fields, 2014, v. 20, № 3, p. 452478.

8. Belopolskaya Ya. Probabilistic counterparts for strongly coupled parabolic systems. In: Springer Proc. Math. Stat. Topics in Statistical Simulation, 2014, v. 114, p. 33-42.

9. Белопольская Я. И. Вероятностная модель системы Лотка-Вольтерра с кроссдиффузией. - Зап. научн. сем. ПОМИ, 2014, v. 431, р. 9-36.

10. Kunita H. Stochastic flows and stochastic differential equations. Cambridge: Cambridge Univ. Press, 1990.

11. Kunita H. Stochastic Flows Acting on Schwartz Distributions. - J. Theor. Pobab., 1994, v. 7, № 2, p. 247-278.

12. Kunita $H$. Generalized solutions of stochastic partial differential equations. - J. Theor. Pobab., 1994, v. 7, № 2, p. 279-308.

13. Белопольская Я. И., Далецкий Ю. Л. Исследование задачи Коши для систем квазилинейных уравнений помощью марковских процессов. - Изв. ВУЗ Математика, 1978 , v. 12 , с. 6-17.

14. Belopolskaya Ya., Dalecky Yu. Stochastic equations and differential geometry. Norwell, MA: Kluwer Academic Publishers, 1990.

15. Belopolskaya Ya., Woyczynski $W$. Generalized solutions of nonlinear parabolic equations and diffusion processes. - Acta Appl. Math., 2007, v. 96, № 1-3, p. 55-69.

16. Belopolskaya Ya., Woyczynski W. Generalized solution of the Cauchy problem for systems of nonlinear parabolic equations and diffusion processes. - Stochast. Dynam., 2012, v. 11, № 1, p. 1-31.

17. Galiano G., Selgas V. On a cross-diffusion segregation problem arising from a model of interacting particles. - Nonlinear Anal., 2014, v. 18, p. 34-49.

18. Fontbona J., Meleard S. Non local Lotka-Volterra system with cross-diffusion in an heterogeneous medium. - J. Math. Biology, 2015, v. 70, № 4, p. 829-854.

19. Protter P. Stochastic Integration and Differential Equations. Berlin: Springer, 2010. 